\title{
NUMERICAL ANALYSIS OF A BDF2 MODULAR GRAD-DIV STABILIZATION METHOD FOR THE NAVIER-STOKES EQUATIONS
}

\author{
Y. RONG* AND J. A. FIORDILINO ${ }^{\dagger}$
}

\begin{abstract}
A second-order accurate modular algorithm is presented for a standard BDF2 code for the Navier-Stokes equations (NSE). The algorithm exhibits resistance to solver breakdown and increased computational efficiency for increasing values of grad-div parameters. We provide a complete theoretical analysis of the algorithms stability and convergency. Computational tests are performed and illustrate the theory and advantages over monolithic grad-div stabilizations.
\end{abstract}

1. Introduction. A common, powerful tool for improving solution quality for fluid flow problems is grad-div stabilization [12, 22, 25, 27, 28. This technique typically involves adding $\gamma \nabla \nabla \cdot u_{h}$, nonzero for most finite element velocity-pressure pairs, which penalizes mass conservation and improves solution accuracy. It was first introduced in [16 and has been widely studied since, both analytically and computationally [4, 12, 20, 22, 23, 26, 27, 28.

Unfortunately, grad-div stabilization also exhibits increased coupling in the linear system's matrix, efficiency loss and solver breakdown, and classical Poisson locking [2, 9, 10, 21, 25, 26, 27. In particular, since the matrix arising from grad-div term is singular, large grad-div parameter $\gamma$ values can cause solver breakdown [8]. This difficulty cannot always be circumvented since recommended parameter choices vary greatly, e.g., from $\mathcal{O}\left(h^{2}\right)$ to $\mathcal{O}\left(10^{4}\right)$ for different applications, finite elements, and meshes [4, 12, 15, 28, 30. An alternate realization of grad-div stabilization with greater computational efficiency was introduced in 5 for the backward Euler time discretization. Herein, we show how to implement modular grad-div stabilization for any multistep time discretization and perform analysis and testing for the BDF2 case.

To begin, consider the incompressible time-dependent NSE: Find the fluid velocity $u$ : $\Omega \times[0, T] \rightarrow \mathbb{R}^{d}$ and pressure $p: \Omega \times(0, T] \rightarrow \mathbb{R}$ satisfying:

$$
\begin{aligned}
& u_{t}+u \cdot \nabla u-\nu \Delta u+\nabla p=f, \text { and } \nabla \cdot u=0 \text { in } \Omega, \\
& u=0 \text { on } \partial \Omega, \text { and } \int_{\Omega} p d x=0, \\
& u(x, 0)=u^{0}(x) \text { in } \Omega .
\end{aligned}
$$

Here, the domain $\Omega \subset \mathbb{R}^{d}(\mathrm{~d}=2,3)$ is a bounded polyhedron, $f$ is the body force and $\nu$ is the fluid viscosity. Suppressing the spacial discretization for the moment, we consider the following two step method that uncouples the grad-div solve.

Step 1: Given $u^{n-1}, u^{n}$, find $\hat{u}^{n+1}$ and $p^{n+1}$ satisfying:

$$
\begin{gathered}
\frac{3 \hat{u}^{n+1}-4 u^{n}+u^{n-1}}{2 \Delta t}+\left(2 u^{n}-u^{n-1}\right) \cdot \nabla \hat{u}^{n+1}-\nu \Delta \hat{u}^{n+1}+\nabla p^{n+1}=f^{n+1}, \\
\nabla \cdot \hat{u}^{n+1}=0 .
\end{gathered}
$$

Step 2: Given $\hat{u}^{n+1}$, find $u^{n+1}$ satisfying:

$$
\frac{3 u^{n+1}-3 \hat{u}^{n+1}}{2 \Delta t}-\beta \nabla \nabla \cdot \frac{3 u^{n+1}-4 u^{n}+u^{n-1}}{2 \Delta t}-\gamma \nabla \nabla \cdot u^{n+1}=0 .
$$

\footnotetext{
*Xi'an Jiaotong University, School of Mathematics and Statistics, Xi'an, Shaanxi 710049, China. Support from NSFC grants 11171269 and 11571274 and China Scholarship Council grant 201606280154.

${ }^{\dagger}$ University of Pittsburgh, Department of Mathematics, Pittsburgh, PA 15260. The research presented herein was partially supported by NSF grants CBET 1609120 and DMS 1522267 . J.A.F. is supported by the DoD SMART Scholarship.
} 
In the above, $\beta \geq 0$ and $\gamma \geq 0$ are application-dependent grad-div stabilization parameters. The combined effect of Step 1 and Step 2 is a consistent BDF2 time discretization of the following model:

$$
u_{t}-\beta \nabla \nabla \cdot u_{t}-\gamma \nabla \nabla \cdot u+u \cdot \nabla u-\nu \Delta u+\nabla p=f .
$$

In [5], two minimally intrusive, modular algorithms were developed for backward Euler, which implemented grad-div stabilization. These algorithms effectively treated issues resulting from increased coupling and solver breakdown. Although the second steps of each of these algorithms can be used here when $\beta \equiv 0$, they cannot be used when $\beta>0$; that is, the dispersive term [3, 18, 29, associated with $\beta$ demands special attention. In the case $\beta>0$, the timediscretizations in both steps must be consistent with one another. In particular, for the BDFk family of methods:

Step 1 : Find $\hat{u}^{n+1}$ and $p^{n+1}$ satisfying:

$$
\begin{gathered}
\frac{1}{\Delta t}\left(a_{0} \hat{u}^{n+1}+\sum_{s=1}^{S} a_{s} u^{n+1-s}\right)+U \cdot \nabla \hat{u}^{n+1}-\nu \Delta \hat{u}^{n+1}+\nabla p^{n+1}=f^{n+1}, \\
\nabla \cdot \hat{u}^{n+1}=0 .
\end{gathered}
$$

Step 2: Find $u^{n+1}$ satisfying:

$$
\frac{a_{0}}{\Delta t}\left(u^{n+1}-\hat{u}^{n+1}\right)-\beta \nabla \nabla \cdot \frac{\sum_{s=0}^{S} a_{s} u^{n+1-s}}{\Delta t}-\gamma \nabla \nabla \cdot u^{n+1}=0,
$$

where $U$ denotes either $\hat{u}^{n+1}$ or a consistent extrapolation. A similar generalization can be made for general linear multistep methods.

This paper is arranged as follows. Section 2 introduces notation, lemmas, and necessary preliminaries. In Section 3, a fully-discrete modular grad-div stabilization algorithm (BDF2$m g d)$ and its unconditional, nonlinear, energy stability are presented. A complete error analysis is given in Section 4 where second-order convergence is proven for the modular method. Numerical experiments are provided to confirm the effectiveness of BDF2-mgd in Section 5 . In particular, the algorithm maintains the positive impact of grad-div stabilization while resisting debilitating slow down for $0 \leq \gamma \leq 20,000$ or $0 \leq \beta \leq 8,000$. Conclusions follow in Section 6 .

2. Preliminaries. We use the standard notations $H^{k}(\Omega), H_{0}^{k}(\Omega)$, and $L^{p}(\Omega)$ to denote Sobolev spaces and $L^{p}$ spaces; see, e.g., [1. The $L^{2}(\Omega)$ inner product and its induced norm are denoted by $(\cdot, \cdot)$ and $\|\cdot\|$, respectively. Let $\|\cdot\|_{L^{p}}$ and $\|\cdot\|_{k}$ denote the $L^{p}(\Omega)(p \neq 2)$ norm and $H^{k}(\Omega)$ norm. The space $H^{-k}(\Omega)$ denotes the dual space of $H_{0}^{k}(\Omega)$ and its norm is denoted by $\|\cdot\|_{-k}$. Throughout the paper, we use $C$ to denote a generic positive constant varying in different places but never depending on mesh size, time step, and grad-div parameters. For functions $v(x, t)$, we define the following norms:

$$
\|v\|_{\infty, k}:=e s s \sup _{[0, T]}\|v(\cdot, t)\|_{k}, \quad\|v\|_{p, k}:=\left(\int_{0}^{T}\|v(\cdot, t)\|_{k}^{p} d t\right)^{\frac{1}{p}}
$$

for $1 \leq p<\infty$. The velocity space $X$, pressure space $Q$, and divergence free space $V$ are defined as follows.

$$
\begin{gathered}
X:=H_{0}^{1}(\Omega)^{d}=\left\{v \in H^{1}(\Omega)^{d}:\left.v\right|_{\partial \Omega}=0\right\}, \\
Q:=L_{0}^{2}(\Omega)=\left\{q \in L^{2}(\Omega): \int_{\Omega} q d x=0\right\}, \\
V:=\{v \in X:(\nabla \cdot v, q)=0 \quad \forall q \in Q\} . \\
2
\end{gathered}
$$


Define the skew-symmetric trilinear form

$$
b(u, v, w):=\frac{1}{2}(u \cdot \nabla v, w)-\frac{1}{2}(u \cdot \nabla w, v) \quad \forall u, v, w \in X .
$$

Then, we have the following estimates for $b$ (see, e.g., Lemma 2.2 in [19]):

$$
\begin{gathered}
b(u, v, w) \leq C\|\nabla u\|\|\nabla v\|\|\nabla w\|, \\
b(u, v, w) \leq C\|u\|^{\frac{1}{2}}\|\nabla u\|^{\frac{1}{2}}\|\nabla v\|\|\nabla w\|, \\
b(u, v, w) \leq C\|u\|\|v\|_{2}\|\nabla w\| .
\end{gathered}
$$

Divide the simulation time $T$ into $N$ smaller time intervals with $[0, T]=\bigcup_{n=0}^{N-1}\left[t^{n}, t^{n+1}\right]$, where $t^{n}=n \Delta t, T=N \Delta t$. We may define the following discrete norms:

$$
\|\| v\left\|\left.\right|_{\infty, k}:=\max _{0 \leq n \leq N}\right\| v\left(\cdot, t^{n}\right)\left\|_{k}, \quad \mid\right\| v \|_{p, k}:=\left(\Delta t \sum_{n=0}^{N}\left\|v\left(\cdot, t^{n}\right)\right\|_{k}^{p}\right)^{\frac{1}{p}} .
$$

Let $\Omega_{h}$ be a quasi-uniform mesh of $\Omega$ with $\bar{\Omega}=\bigcup_{K \in \Omega_{h}} K$. Denote $h=\sup _{K \in \Omega_{h}} \operatorname{diam}(K)$. Let $X_{h} \subset X$ and $Q_{h} \subset Q$ be the finite element spaces. Assume that $X_{h}$ and $Q_{h}$ satisfy approximation properties of piecewise continuous polynomials on quasi-uniform meshes of local degrees $k$ and $m$, respectively:

$$
\begin{array}{lc}
\inf _{v_{h} \in X_{h}}\left\|u-v_{h}\right\| \leq C h^{k+1}|u|_{k+1} & u \in X \cap H^{k+1}(\Omega)^{d}, \\
\inf _{v_{h} \in X_{h}}\left\|u-v_{h}\right\|_{1} \leq C h^{k}|u|_{k+1} & u \in X \cap H^{k+1}(\Omega)^{d}, \\
\inf _{q_{h} \in Q_{h}}\left\|p-q_{h}\right\| \leq C h^{m+1}|p|_{m+1} & p \in Q \cap H^{m+1}(\Omega) .
\end{array}
$$

Furthermore, we assume that $X_{h}$ and $Q_{h}$ satisfy the usual discrete inf-sup condition:

$$
\inf _{q \in Q_{h}} \sup _{v \in X_{h}} \frac{(q, \nabla \cdot v)}{\|\nabla v\|\|q\|} \geq C_{0}>0 .
$$

The discrete divergence-free space $V_{h}$ is defined by

$$
V_{h}:=\left\{v_{h} \in X_{h}:\left(\nabla \cdot v_{h}, q_{h}\right)=0 \quad \forall q_{h} \in Q_{h}\right\} .
$$

Note that the well-known Taylor-Hood mixed finite element is one such example satisfying the above assumptions with $k=2, m=1$.

The following lemmas will be useful in later analyses. For their proofs, see Theorem 1.1 on p. 59 of [7] for Lemma 2.1] Lemma 2 of [24 for Lemma 2.2, and Lemma 5.1 on p. 369 of [1] for Lemma 2.3 .

Lemma 2.1. Suppose that the finite element spaces satisfy 2.7). Then, for any $u \in V$, we have

$$
\inf _{v_{h} \in V_{h}}\left\|\nabla\left(u-v_{h}\right)\right\| \leq C \inf _{v_{h} \in X_{h}}\left\|\nabla\left(u-v_{h}\right)\right\|
$$

Lemma 2.2. If $g_{t}, g_{t t}, g_{t t t} \in L^{2}\left(0, T ; H^{r}(\Omega)\right)$, then we have

$$
\left\|g^{n+1}-2 g^{n}+g^{n-1}\right\|_{r}^{2} \leq C \Delta t^{3} \int_{t^{n-1}}^{t^{n+1}}\left\|g_{t t}\right\|_{r}^{2} d t
$$




$$
\begin{gathered}
\left\|3 g^{n+1}-4 g^{n}+g^{n-1}\right\|_{r}^{2} \leq C \Delta t \int_{t^{n-1}}^{t^{n+1}}\left\|g_{t}\right\|_{r}^{2} d t \\
\left\|\frac{3 g^{n+1}-4 g^{n}+g^{n-1}}{2 \Delta t}-g_{t}\left(t^{n+1}\right)\right\|_{r}^{2} \leq C \Delta t^{3} \int_{t^{n-1}}^{t^{n+1}}\left\|g_{t t t}\right\|_{r}^{2} d t .
\end{gathered}
$$

LEMma 2.3. (The discrete Gronwall's lemma, without $\Delta$ t-restriction) Suppose that $n$ and $N$ are nonnegative integers, $n \leq N$. The real numbers $a_{n}, b_{n}, c_{n}, \kappa_{n}, \Delta t, C$ are nonnegative and satisfy

$$
a_{N}+\Delta t \sum_{n=0}^{N} b_{n} \leq \Delta t \sum_{n=0}^{N-1} \kappa_{n} a_{n}+\Delta t \sum_{n=0}^{N} c_{n}+C .
$$

Then,

$$
a_{N}+\Delta t \sum_{n=0}^{N} b_{n} \leq \exp \left(\Delta t \sum_{n=0}^{N-1} \kappa_{n}\right)\left(\Delta t \sum_{n=0}^{N} c_{n}+C\right) .
$$

3. The BDF2 modular grad-div stabilization algorithm and its stability. We propose the following fully-discrete modular grad-div stabilization algorithm for approximating solutions of (1.1).

BDF2-mgd:

Step 1: Given $u_{h}^{n-1}, u_{h}^{n} \in X_{h}$, find $\left(\hat{u}_{h}^{n+1}, p_{h}^{n+1}\right) \in\left(X_{h}, Q_{h}\right)$ satisfying:

$$
\begin{gathered}
\left(\frac{3 \hat{u}_{h}^{n+1}-4 u_{h}^{n}+u_{h}^{n-1}}{2 \Delta t}, v_{h}\right)+b\left(2 u_{h}^{n}-u_{h}^{n-1}, \hat{u}_{h}^{n+1}, v_{h}\right)+\nu\left(\nabla \hat{u}_{h}^{n+1}, \nabla v_{h}\right) \\
-\left(p_{h}^{n+1}, \nabla \cdot v_{h}\right)=\left(f^{n+1}, v_{h}\right) \quad \forall v_{h} \in X_{h}, \\
\left(\nabla \cdot \hat{u}_{h}^{n+1}, q_{h}\right)=0 \quad \forall q_{h} \in Q_{h} .
\end{gathered}
$$

Step 2: Given $\hat{u}_{h}^{n+1} \in X_{h}$, find $u_{h}^{n+1} \in X_{h}$ satisfying:

$$
\begin{gathered}
\left(\frac{3 u_{h}^{n+1}-3 \hat{u}_{h}^{n+1}}{2 \Delta t}, v_{h}\right)+\beta\left(\nabla \cdot \frac{3 u_{h}^{n+1}-4 u_{h}^{n}+u_{h}^{n-1}}{2 \Delta t}, \nabla \cdot v_{h}\right) \\
+\gamma\left(\nabla \cdot u_{h}^{n+1}, \nabla \cdot v_{h}\right)=0 \quad \forall v_{h} \in X_{h} .
\end{gathered}
$$

Remark 1. When $\beta=0$, Step 2 is equivalent to Step 2 appearing in [5] with $\gamma \leftarrow \frac{2}{3} \gamma$.

Step 2 of BDF2-mgd appears to be overdetermined since both the tangential and normal components of the solution are prescribed on the boundary. However, due to the zeroth-order term, it is not; a unique solution always exists, Theorem 3.1. and converges to the true NSE solution, Theorems 4.4 and 4.5

TheOREm 3.1. Suppose $f^{n+1} \in H^{-1}(\Omega)^{d}$ and $u_{h}^{n-1}, u_{h}^{n} \in X_{h}$. Then, there exists unique solutions $\hat{u}_{h}^{n+1}, u_{h}^{n+1} \in X_{h}$ to BDF2-mgd.

Proof. The proof follows by similar arguments as in Theorem 5 of [5].

Next, we analyze the stability of BDF2-mgd. We first prove an important lemma for the stability analysis. Unconditional, nonlinear, energy stability is then proven in Theorem 3.3

LEmma 3.2. Consider BDF2-mgd, then the following identities hold for Step 2 (3.3):

$$
\begin{aligned}
\left\|\hat{u}_{h}^{n+1}\right\|^{2}= & \left\|u_{h}^{n+1}\right\|^{2}+\left\|\hat{u}_{h}^{n+1}-u_{h}^{n+1}\right\|^{2}+\frac{4}{3} \gamma \Delta t\left\|\nabla \cdot u_{h}^{n+1}\right\|^{2} \\
& +\frac{\beta}{3}\left(\left\|\nabla \cdot u_{h}^{n+1}\right\|^{2}-\left\|\nabla \cdot u_{h}^{n}\right\|^{2}+\left\|\nabla \cdot\left(2 u_{h}^{n+1}-u_{h}^{n}\right)\right\|^{2}\right. \\
& \left.-\left\|\nabla \cdot\left(2 u_{h}^{n}-u_{h}^{n-1}\right)\right\|^{2}+\left\|\nabla \cdot\left(u_{h}^{n+1}-2 u_{h}^{n}+u_{h}^{n-1}\right)\right\|^{2}\right),
\end{aligned}
$$


and

$$
\begin{aligned}
& \left(\frac{3 u_{h}^{n+1}-4 u_{h}^{n}+u_{h}^{n-1}}{2 \Delta t}, \hat{u}_{h}^{n+1}-u_{h}^{n+1}\right) \\
& =\frac{\beta}{6 \Delta t}\left\|\nabla \cdot\left(3 u_{h}^{n+1}-4 u_{h}^{n}+u_{h}^{n-1}\right)\right\|^{2}+\frac{\gamma}{3}\left(\nabla \cdot u_{h}^{n+1}, \nabla \cdot\left(3 u_{h}^{n+1}-4 u_{h}^{n}+u_{h}^{n-1}\right)\right) .
\end{aligned}
$$

Proof. Selecting $v_{h}=\frac{4 \Delta t}{3} u_{h}^{n+1}$ in 3.3 , we have

$$
\begin{aligned}
& 2\left\|u_{h}^{n+1}\right\|^{2}-2\left(\hat{u}_{h}^{n+1}, u_{h}^{n+1}\right)+\frac{4}{3} \gamma \Delta t\left\|\nabla \cdot u_{h}^{n+1}\right\|^{2}+\frac{\beta}{3}\left(\left\|\nabla \cdot u_{h}^{n+1}\right\|^{2}-\left\|\nabla \cdot u_{h}^{n}\right\|^{2}\right. \\
& \left.+\left\|\nabla \cdot\left(2 u_{h}^{n+1}-u_{h}^{n}\right)\right\|^{2}-\left\|\nabla \cdot\left(2 u_{h}^{n}-u_{h}^{n-1}\right)\right\|^{2}+\left\|\nabla \cdot\left(u_{h}^{n+1}-2 u_{h}^{n}+u_{h}^{n-1}\right)\right\|^{2}\right)=0
\end{aligned}
$$

where we have used the identity $2(3 a-4 b+c) a=a^{2}-b^{2}+(2 a-b)^{2}-(2 b-c)^{2}+(a-2 b+c)^{2}$ on the third term. For the second term in (3.6), using the following polarization identity

$$
\left(\hat{u}_{h}^{n+1}, u_{h}^{n+1}\right)=\frac{1}{2}\left\|\hat{u}_{h}^{n+1}\right\|^{2}+\frac{1}{2}\left\|u_{h}^{n+1}\right\|^{2}-\frac{1}{2}\left\|\hat{u}_{h}^{n+1}-u_{h}^{n+1}\right\|^{2}
$$

yields the first identity 3.4 . The second follows by setting $v_{h}=\frac{3 u_{h}^{n+1}-4 u_{h}^{n}+u_{h}^{n-1}}{3}$ in 3.3 . We are now in a position to prove unconditional stability.

TheOREm 3.3. Suppose $f \in L^{2}\left(0, T ; H^{-1}(\Omega)^{d}\right)$, then the following holds for all $N \geq 1$.

$$
\begin{aligned}
& \left\|u_{h}^{N}\right\|^{2}+\left\|2 u_{h}^{N}-u_{h}^{N-1}\right\|^{2}+\left(\frac{2 \gamma \Delta t}{3}+\beta\right)\left\|\nabla \cdot u_{h}^{N}\right\|^{2}+\left(\frac{2 \gamma \Delta t}{3}+\beta\right)\left\|\nabla \cdot\left(2 u_{h}^{N}-u_{h}^{N-1}\right)\right\|^{2} \\
& \quad+4 \gamma \Delta t \sum_{n=1}^{N-1}\left\|\nabla \cdot u_{h}^{n+1}\right\|^{2}+2 \nu \Delta t \sum_{n=1}^{N-1}\left\|\nabla \hat{u}_{h}^{n+1}\right\|^{2} \\
& \leq \frac{2 \Delta t}{\nu} \sum_{n=1}^{N-1}\left\|f^{n+1}\right\|_{-1}^{2}+\left\|u_{h}^{1}\right\|^{2}+\left\|2 u_{h}^{1}-u_{h}^{0}\right\|^{2} \\
& \quad+\left(\frac{2 \gamma \Delta t}{3}+\beta\right)\left\|\nabla \cdot u_{h}^{1}\right\|^{2}+\left(\frac{2 \gamma \Delta t}{3}+\beta\right)\left\|\nabla \cdot\left(2 u_{h}^{1}-u_{h}^{0}\right)\right\|^{2} .
\end{aligned}
$$

Proof. Set $v_{h}=\hat{u}_{h}^{n+1}$ in 3.1 and $q_{h}=p_{h}^{n+1}$ in 3.2. Adding these two equations and rearranging the discrete time derivative yields

$$
\begin{aligned}
& \left(\frac{3 u_{h}^{n+1}-4 u_{h}^{n}+u_{h}^{n-1}}{2 \Delta t}, u_{h}^{n+1}\right)+\left(\frac{3 u_{h}^{n+1}-4 u_{h}^{n}+u_{h}^{n-1}}{2 \Delta t}, \hat{u}_{h}^{n+1}-u_{h}^{n+1}\right) \\
& +\left(\frac{3 \hat{u}_{h}^{n+1}-3 u_{h}^{n+1}}{2 \Delta t}, \hat{u}_{h}^{n+1}\right)+\nu\left\|\nabla \hat{u}_{h}^{n+1}\right\|^{2}=\left(f^{n+1}, \hat{u}_{h}^{n+1}\right) .
\end{aligned}
$$

Consider the resulting time derivative terms. Use the identity $2(3 a-4 b+c) a=a^{2}-b^{2}+(2 a-$ $b)^{2}-(2 b-c)^{2}+(a-2 b+c)^{2}$ on the first term and both $(3.5)$ of Lemma 3.2 and the identity on the second term. Apply the polarization identity to the third term. Then,

$$
\begin{aligned}
& \frac{1}{4 \Delta t}\left(\left\|u_{h}^{n+1}\right\|^{2}-\left\|u_{h}^{n}\right\|^{2}+\left\|2 u_{h}^{n+1}-u_{h}^{n}\right\|^{2}-\left\|2 u_{h}^{n}-u_{h}^{n-1}\right\|^{2}+\left\|u_{h}^{n+1}-2 u_{h}^{n}+u_{h}^{n-1}\right\|^{2}\right) \\
& \quad+\frac{\gamma}{6}\left(\left\|\nabla \cdot u_{h}^{n+1}\right\|^{2}-\left\|\nabla \cdot u_{h}^{n}\right\|^{2}+\left\|\nabla \cdot\left(2 u_{h}^{n+1}-u_{h}^{n}\right)\right\|^{2}-\left\|\nabla \cdot\left(2 u_{h}^{n}-u_{h}^{n-1}\right)\right\|^{2}\right. \\
& \left.\quad+\left\|\nabla \cdot\left(u_{h}^{n+1}-2 u_{h}^{n}+u_{h}^{n-1}\right)\right\|^{2}\right)+\frac{\beta}{6 \Delta t}\left\|\nabla \cdot\left(3 u_{h}^{n+1}-4 u_{h}^{n}+u_{h}^{n-1}\right)\right\|^{2} \\
& \quad+\frac{3}{4 \Delta t}\left(\left\|\hat{u}_{h}^{n+1}\right\|^{2}-\left\|u_{h}^{n+1}\right\|^{2}+\left\|\hat{u}_{h}^{n+1}-u_{h}^{n+1}\right\|^{2}\right)+\nu\left\|\nabla \hat{u}_{h}^{n+1}\right\|^{2} \\
& =\left(f^{n+1}, \hat{u}_{h}^{n+1}\right) .
\end{aligned}
$$


Multiply (3.10) by $4 \Delta t$ and use (3.4) of Lemma 3.2. Then

$$
\begin{aligned}
& \left\|u_{h}^{n+1}\right\|^{2}-\left\|u_{h}^{n}\right\|^{2}+\left\|2 u_{h}^{n+1}-u_{h}^{n}\right\|^{2}-\left\|2 u_{h}^{n}-u_{h}^{n-1}\right\|^{2}+\left\|u_{h}^{n+1}-2 u_{h}^{n}+u_{h}^{n-1}\right\|^{2} \\
& \quad+\frac{2 \gamma \Delta t}{3}\left(\left\|\nabla \cdot u_{h}^{n+1}\right\|^{2}-\left\|\nabla \cdot u_{h}^{n}\right\|^{2}+\left\|\nabla \cdot\left(2 u_{h}^{n+1}-u_{h}^{n}\right)\right\|^{2}-\left\|\nabla \cdot\left(2 u_{h}^{n}-u_{h}^{n-1}\right)\right\|^{2}\right. \\
& \left.\quad+\left\|\nabla \cdot\left(u_{h}^{n+1}-2 u_{h}^{n}+u_{h}^{n-1}\right)\right\|^{2}\right)+\frac{2 \beta}{3}\left\|\nabla \cdot\left(3 u_{h}^{n+1}-4 u_{h}^{n}+u_{h}^{n-1}\right)\right\|^{2} \\
& \quad+\beta\left(\left\|\nabla \cdot u_{h}^{n+1}\right\|^{2}-\left\|\nabla \cdot u_{h}^{n}\right\|^{2}+\left\|\nabla \cdot\left(2 u_{h}^{n+1}-u_{h}^{n}\right)\right\|^{2}-\left\|\nabla \cdot\left(2 u_{h}^{n}-u_{h}^{n-1}\right)\right\|^{2}\right. \\
& \left.\quad+\left\|\nabla \cdot\left(u_{h}^{n+1}-2 u_{h}^{n}+u_{h}^{n-1}\right)\right\|^{2}\right)+6\left\|\hat{u}_{h}^{n+1}-u_{h}^{n+1}\right\|^{2} \\
& \quad+4 \gamma \Delta t\left\|\nabla \cdot u_{h}^{n+1}\right\|^{2}+4 \nu \Delta t\left\|\nabla \hat{u}_{h}^{n+1}\right\|^{2} \\
& =4 \Delta t\left(f^{n+1}, \hat{u}_{h}^{n+1}\right) .
\end{aligned}
$$

Summing 3.11 from $n=1$ to $N-1$ yields

$$
\begin{aligned}
& \left\|u_{h}^{N}\right\|^{2}+\left\|2 u_{h}^{N}-u_{h}^{N-1}\right\|^{2}+\frac{2 \gamma \Delta t}{3}\left\|\nabla \cdot u_{h}^{N}\right\|^{2}+\frac{2 \gamma \Delta t}{3}\left\|\nabla \cdot\left(2 u_{h}^{N}-u_{h}^{N-1}\right)\right\|^{2}+\beta\left\|\nabla \cdot u_{h}^{N}\right\|^{2} \\
& \quad+\beta\left\|\nabla \cdot\left(2 u_{h}^{N}-u_{h}^{N-1}\right)\right\|^{2}+4 \gamma \Delta t \sum_{n=1}^{N-1}\left\|\nabla \cdot u_{h}^{n+1}\right\|^{2}+4 \nu \Delta t \sum_{n=1}^{N-1}\left\|\nabla \hat{u}_{h}^{n+1}\right\|^{2} \\
& \leq 4 \Delta t \sum_{n=1}^{N-1}\left(f^{n+1}, \hat{u}_{h}^{n+1}\right)+\left\|u_{h}^{1}\right\|^{2}+\left\|2 u_{h}^{1}-u_{h}^{0}\right\|^{2} \\
& \quad+\frac{2 \gamma \Delta t}{3}\left\|\nabla \cdot u_{h}^{1}\right\|^{2}+\frac{2 \gamma \Delta t}{3}\left\|\nabla \cdot\left(2 u_{h}^{1}-u_{h}^{0}\right)\right\|^{2}+\beta\left\|\nabla \cdot u_{h}^{1}\right\|^{2}+\beta\left\|\nabla \cdot\left(2 u_{h}^{1}-u_{h}^{0}\right)\right\|^{2} .
\end{aligned}
$$

Finally, using the Cauchy-Schwarz-Young inequality on the first term on the right hand side completes the proof.

Remark 2. Lemma 3.2 and Theorem 3.3 imply stability of $\hat{u}_{h}$ with respect to $|\|\cdot\||_{\infty, 0}$.

4. Error Analysis. In this section, we provide á priori error estimates for BDF2-mgd. In particular, we show that BDF2-mgd is second-order convergent. Denote $u^{n}=u\left(t^{n}\right)$ for $n=0,1, \cdots, N$ (and similarly for all other variables). The errors are denoted by

$$
e_{u}^{n}=u^{n}-u_{h}^{n}, \quad e_{\hat{u}}^{n}=u^{n}-\hat{u}_{h}^{n}, \quad e_{p}^{n}=p^{n}-p_{h}^{n+1} .
$$

Decompose the velocity errors

$$
\begin{aligned}
& e_{u}^{n}=\eta^{n}-\phi_{h}^{n}, \quad \eta^{n}:=u^{n}-\tilde{u}^{n}, \quad \phi_{h}^{n}:=u_{h}^{n}-\tilde{u}^{n}, \\
& e_{\hat{u}}^{n}=\eta^{n}-\psi_{h}^{n}, \quad \psi_{h}^{n}:=\hat{u}_{h}^{n}-\tilde{u}^{n},
\end{aligned}
$$

where $\tilde{u}^{n}$ denotes an interpolant of $u^{n}$ in $V_{h}$.

Definition 4.1. Define the following consistency errors. For all $v_{h} \in V_{h}$,

$$
\tau^{n+1}\left(v_{h}\right):=\left(\frac{3 u^{n+1}-4 u^{n}+u^{n-1}}{2 \Delta t}-u_{t}^{n+1}, v_{h}\right)-b\left(u^{n+1}-2 u^{n}+u^{n-1}, u^{n+1}, v_{h}\right) .
$$

Lemma 4.2. Assume the true solution u satisfies the following,

$$
u \in L^{\infty}\left(0, T ; H^{1}(\Omega)^{d}\right), u_{t t} \in L^{2}\left(0, T ; H^{1}(\Omega)^{d}\right), u_{t t t} \in L^{2}\left(0, T ; H^{-1}(\Omega)^{d}\right) .
$$

Then, $\forall \sigma>0$, we have

$$
\left|\tau^{n+1}\left(v_{h}\right)\right| \leq \frac{C}{2 \sigma} \Delta t^{3}\left(\int_{t^{n-1}}^{t^{n+1}}\left\|u_{t t t}\right\|_{-1}^{2} d t+\int_{t^{n-1}}^{t^{n+1}}\left\|\nabla u_{t t}\right\|^{2} d t\right)+\sigma\left\|\nabla v_{h}\right\|^{2} .
$$


Proof. For an arbitrary $\sigma>0$,

$$
\begin{aligned}
\left|\tau^{n+1}\left(v_{h}\right)\right| & \\
\leq & \left\|\frac{3 u^{n+1}-4 u^{n}+u^{n-1}}{2 \Delta t}-u_{t}^{n+1}\right\|_{-1}\left\|\nabla v_{h}\right\|+C\left\|\nabla\left(u^{n+1}-2 u^{n}+u^{n-1}\right)\right\|\left\|\nabla u^{n+1}\right\|\left\|\nabla v_{h}\right\| \\
\leq & \frac{1}{2 \sigma}\left\|\frac{3 u^{n+1}-4 u^{n}+u^{n-1}}{2 \Delta t}-u_{t}^{n+1}\right\|_{-1}^{2} \\
& +\frac{C}{2 \sigma}\left\|\nabla u^{n+1}\right\|^{2}\left\|\nabla\left(u^{n+1}-2 u^{n}+u^{n-1}\right)\right\|^{2}+\sigma\left\|\nabla v_{h}\right\|^{2} \\
\leq & \frac{C}{2 \sigma} \Delta t^{3}\left(\int_{t^{n-1}}^{t^{n+1}}\left\|u_{t t t}\right\|_{-1}^{2} d t+\int_{t^{n-1}}^{t^{n+1}}\left\|\nabla u_{t t}\right\|^{2} d t\right)+\sigma\left\|\nabla v_{h}\right\|^{2},
\end{aligned}
$$

where we use the Cauchy-Schwarz-Young inequality and Lemma 2.2. $\mathrm{x}$

Once again, we require a key lemma, regarding Step 2, to prove convergence.

LEMma 4.3. The following inequality holds.

$$
\begin{aligned}
\left\|\psi_{h}^{n+1}\right\|^{2} \geq & \left\|\phi_{h}^{n+1}\right\|^{2}+\left\|\phi_{h}^{n+1}-\psi_{h}^{n+1}\right\|^{2}+\frac{\beta}{3}\left(\left\|\nabla \cdot \phi_{h}^{n+1}\right\|^{2}-\left\|\nabla \cdot \phi_{h}^{n}\right\|^{2}+\left\|\nabla \cdot\left(2 \phi_{h}^{n+1}-\phi_{h}^{n}\right)\right\|^{2}\right. \\
& \left.-\left\|\nabla \cdot\left(2 \phi_{h}^{n}-\phi_{h}^{n-1}\right)\right\|^{2}+\frac{1}{2}\left\|\nabla \cdot\left(\phi_{h}^{n+1}-2 \phi_{h}^{n}+\phi_{h}^{n-1}\right)\right\|^{2}\right)+\frac{2 \gamma \Delta t}{3}\left\|\nabla \cdot \phi_{h}^{n+1}\right\|^{2}(4.5) \\
& -\frac{C \beta d(1+2 \Delta t)}{3} \int_{t^{n-1}}^{t^{n+1}}\left\|\nabla \eta_{t}\right\|^{2} d t-\frac{\beta \Delta t}{3}\left\|\nabla \cdot\left(2 \phi_{h}^{n}-\phi_{h}^{n-1}\right)\right\|-\frac{2 \gamma d \Delta t}{3}\left\|\nabla \eta^{n+1}\right\|^{2} .
\end{aligned}
$$

Proof. At time $t^{n+1}$, for all $v_{h} \in X_{h}$, the true solution $u$ satisfies

$$
\left(\frac{3 u^{n+1}-3 u^{n+1}}{2 \Delta t}, v_{h}\right)+\beta\left(\nabla \cdot \frac{3 u^{n+1}-4 u^{n}+u^{n-1}}{2 \Delta t}, \nabla \cdot v_{h}\right)+\gamma\left(\nabla \cdot u^{n+1}, \nabla \cdot v_{h}\right)=0 .
$$

Subtracting (4.6) from 3.3 , we have

$$
\left(\frac{3 e_{u}^{n+1}-3 e_{\hat{u}}^{n+1}}{2 \Delta t}, v_{h}\right)+\beta\left(\nabla \cdot \frac{3 e_{u}^{n+1}-4 e_{u}^{n}+e_{u}^{n-1}}{2 \Delta t}, \nabla \cdot v_{h}\right)+\gamma\left(\nabla \cdot e_{u}^{n+1}, \nabla \cdot v_{h}\right)=0
$$

Setting $v_{h}=\phi_{h}^{n+1}$ in 4.7 , using similar identities as in Theorem 3.2 and rearranging terms yields

$$
\begin{aligned}
\left\|\psi_{h}^{n+1}\right\|^{2}= & \left\|\phi_{h}^{n+1}\right\|^{2}+\left\|\phi_{h}^{n+1}-\psi_{h}^{n+1}\right\|^{2}+\frac{\beta}{3}\left(\left\|\nabla \cdot \phi_{h}^{n+1}\right\|^{2}-\left\|\nabla \cdot \phi_{h}^{n}\right\|^{2}+\left\|\nabla \cdot\left(2 \phi_{h}^{n+1}-\phi_{h}^{n}\right)\right\|^{2}\right. \\
& \left.-\left\|\nabla \cdot\left(2 \phi_{h}^{n}-\phi_{h}^{n-1}\right)\right\|^{2}+\left\|\nabla \cdot\left(\phi_{h}^{n+1}-2 \phi_{h}^{n}+\phi_{h}^{n-1}\right)\right\|^{2}\right)+\frac{4 \gamma \Delta t}{3}\left\|\nabla \cdot \phi_{h}^{n+1}\right\|^{2} \quad(4.8) \\
& -\frac{2 \beta}{3}\left(\nabla \cdot\left(3 \eta^{n+1}-4 \eta^{n}+\eta^{n-1}\right), \nabla \cdot \phi_{h}^{n+1}\right)-\frac{4 \gamma \Delta t}{3}\left(\nabla \cdot \eta^{n+1}, \nabla \cdot \phi_{h}^{n+1}\right) .
\end{aligned}
$$

Split $-\frac{2 \beta}{3}\left(\nabla \cdot\left(3 \eta^{n+1}-4 \eta^{n}+\eta^{n-1}\right), \nabla \cdot \phi_{h}^{n+1}\right)$ into $-\frac{2 \beta}{3}\left(\nabla \cdot\left(3 \eta^{n+1}-4 \eta^{n}+\eta^{n-1}\right), \nabla \cdot\left(\phi_{h}^{n+1}-\right.\right.$ $\left.\left.2 \phi_{h}^{n}+\phi_{h}^{n-1}\right)\right)-\frac{2 \beta}{3}\left(\nabla \cdot\left(3 \eta^{n+1}-4 \eta^{n}+\eta^{n-1}\right), \nabla \cdot\left(2 \phi_{h}^{n}-\phi_{h}^{n-1}\right)\right)$. Using the Cauchy-Schwarz-Young 
inequality and Lemma 2.2. Then, the following three inequalities hold,

$$
\begin{aligned}
& \left|\frac{2 \beta}{3}\left(\nabla \cdot\left(3 \eta^{n+1}-4 \eta^{n}+\eta^{n-1}\right), \nabla \cdot\left(2 \phi_{h}^{n}-\phi_{h}^{n-1}\right)\right)\right| \\
& \leq \frac{2 \beta \sqrt{d}}{3}\left\|\nabla\left(3 \eta^{n+1}-4 \eta^{n}+\eta^{n-1}\right)\right\|\left\|\nabla \cdot\left(2 \phi_{h}^{n}-\phi_{h}^{n-1}\right)\right\| \\
& \leq \frac{C \beta d}{3} \int_{t^{n-1}}^{t^{n+1}}\left\|\nabla \eta_{t}\right\|^{2} d t+\frac{\beta \Delta t}{3}\left\|\nabla \cdot\left(2 \phi_{h}^{n}-\phi_{h}^{n-1}\right)\right\|^{2} \\
& \leq \frac{C \beta d}{3} \int_{t^{n-1}}^{t^{n+1}}\left\|\nabla \eta_{t}\right\|^{2} d t+\frac{\beta \Delta t}{3}\left(\left\|\nabla \cdot\left(2 \phi_{h}^{n}-\phi_{h}^{n-1}\right)\right\|^{2}+\left\|\nabla \cdot \phi_{h}^{n}\right\|^{2}\right), \\
& \quad\left|\frac{2 \beta}{3}\left(\nabla \cdot\left(3 \eta^{n+1}-4 \eta^{n}+\eta^{n-1}\right), \nabla \cdot\left(\phi_{h}^{n+1}-2 \phi_{h}^{n}+\phi_{h}^{n-1}\right)\right)\right| \\
& \quad \leq \frac{2 \beta \sqrt{d}}{3}\left\|\nabla\left(3 \eta^{n+1}-4 \eta^{n}+\eta^{n-1}\right)\right\|\left\|\nabla \cdot\left(\phi_{h}^{n+1}-2 \phi_{h}^{n}+\phi_{h}^{n-1}\right)\right\| \\
& \quad \leq \frac{2 C \beta d \Delta t}{3} \int_{t^{n-1}}^{t^{n+1}}\left\|\nabla \eta_{t}\right\|^{2} d t+\frac{\beta}{6}\left\|\nabla \cdot\left(\phi_{h}^{n+1}-2 \phi_{h}^{n}+\phi_{h}^{n-1}\right)\right\|^{2},
\end{aligned}
$$

and

$$
\begin{aligned}
\left|\frac{4 \gamma \Delta t}{3}\left(\nabla \cdot \eta^{n+1}, \nabla \cdot \phi_{h}^{n+1}\right)\right| & \leq \frac{4 \gamma \sqrt{d} \Delta t}{3}\left\|\nabla \eta^{n+1}\right\|\left\|\nabla \cdot \phi_{h}^{n+1}\right\| \\
& \leq \frac{2 \gamma d \Delta t}{3}\left\|\nabla \eta^{n+1}\right\|^{2}+\frac{2 \gamma \Delta t}{3}\left\|\nabla \cdot \phi_{h}^{n+1}\right\| .
\end{aligned}
$$

Combining 4.8 - 4.11) completes the proof. $\square$

Next, we give the main error result for BDF2-mgd when $\beta>0$.

TheOrem 4.4. Assume the true solution $u, p$ satisfy (4.2) and the following regularity

$$
\begin{aligned}
& u \in L^{\infty}\left(0, T ; H^{k+1}(\Omega)^{d}\right) \cap L^{2}\left(0, T ; H^{k+1}(\Omega)^{d}\right), \\
& u_{t} \in L^{2}\left(0, T ; H^{k+1}(\Omega)^{d}\right), \quad p \in L^{2}\left(0, T ; H^{m+1}(\Omega)\right) .
\end{aligned}
$$

Then, we have the following estimates for BDF2-mgd.

$$
\begin{aligned}
& \left\|e_{u}^{N}\right\|^{2}+\left\|2 e_{u}^{N}-e_{u}^{N-1}\right\|^{2}+\left(\frac{2 \gamma \Delta t}{3}+\beta\right)\left(\left\|\nabla \cdot e_{u}^{N}\right\|^{2}+\left\|\nabla \cdot\left(2 e_{u}^{N}-e_{u}^{N-1}\right)\right\|^{2}\right) \\
& \quad+2 \nu \Delta t \sum_{n=1}^{N-1}\left\|\nabla e_{\hat{u}}^{n+1}\right\|^{2}+2 \gamma \Delta t \sum_{n=1}^{N-1}\left\|\nabla \cdot e_{u}^{n+1}\right\|^{2} \\
& \leq \\
& \quad C \exp \left(C^{*} T\right)\left\{\operatorname { i n f } _ { v _ { h } \in X _ { h } } \left(\beta(1+\Delta t)\left\|\nabla\left(u-v_{h}\right)_{t}\right\|_{2,0}^{2}+\frac{1}{\nu}\left\|\left(u-v_{h}\right)_{t}\right\|_{2,0}^{2}\right.\right. \\
& \quad+\left(\frac{\gamma^{2} \Delta t}{\beta}+\gamma+\nu+\frac{1}{\nu}\right) \mid\left\|\nabla\left(u-v_{h}\right)\right\|_{2,0}^{2}+\left(\frac{2 \gamma \Delta t}{3}+\beta+\frac{1}{\nu^{2}}\right)\left\|\nabla\left(u-v_{h}\right)\right\|_{\infty, 0}^{2} \\
& \left.\quad+\left\|u-v_{h}\right\|_{\infty, 0}^{2}\right)+\frac{1}{\nu} \inf _{q_{h} \in Q_{h}} \mid\left\|p-q_{h}\right\|_{2,0}^{2}+\frac{1}{\nu} \Delta t^{4} \\
& \left.\quad+\left\|e_{u}^{1}\right\|^{2}+\left\|2 e_{u}^{1}-e_{u}^{0}\right\|^{2}+\left(\frac{2 \gamma \Delta t}{3}+\beta\right)\left(\left\|\nabla \cdot e_{u}^{1}\right\|^{2}+\left\|\nabla \cdot\left(2 e_{u}^{1}-e_{u}^{0}\right)\right\|^{2}\right)\right\}
\end{aligned}
$$


Proof. At time $t^{n+1}$, the true solution $u, p$ satisfies

$$
\begin{gathered}
\left(\frac{3 u^{n+1}-4 u^{n}+u^{n-1}}{2 \Delta t}, v_{h}\right)+b\left(2 u^{n}-u^{n-1}, u^{n+1}, v_{h}\right)+\nu\left(\nabla u^{n+1}, \nabla v_{h}\right) \\
-\left(p^{n+1}, \nabla \cdot v_{h}\right)=\left(f^{n+1}, v_{h}\right)+\tau^{n+1}\left(v_{h}\right) \quad \forall v_{h} \in X_{h}, \\
\left(\nabla \cdot u^{n+1}, q_{h}\right)=0 \quad \forall q_{h} \in Q_{h} .
\end{gathered}
$$

Subtracting (3.1) and $(3.2)$ from (4.14) and 4.15), respectively, we have

$$
\begin{gathered}
\left(\frac{3 e_{\hat{u}}^{n+1}-4 e_{u}^{n}+e_{u}^{n-1}}{2 \Delta t}, v_{h}\right)+b\left(2 u^{n}-u^{n-1}, u^{n+1}, v_{h}\right)-b\left(2 u_{h}^{n}-u_{h}^{n-1}, \hat{u}_{h}^{n+1}, v_{h}\right) \\
+\nu\left(\nabla e_{\hat{u}}^{n+1}, \nabla v_{h}\right)-\left(e_{p}^{n+1}, \nabla \cdot v_{h}\right)=\tau^{n+1}\left(v_{h}\right) \quad \forall v_{h} \in X_{h} \\
\left(\nabla \cdot e_{\hat{u}}^{n+1}, q_{h}\right)=0 \quad \forall q_{h} \in Q_{h} .
\end{gathered}
$$

Set $v_{h}=\psi_{h}^{n+1} \in V_{h}$ in equation 4.16 , then

$$
\begin{aligned}
& \left(\frac{3 \eta^{n+1}-4 \eta^{n}+\eta^{n-1}}{2 \Delta t}, \psi_{h}^{n+1}\right)-\left(\frac{3 \phi_{h}^{n+1}-4 \phi_{h}^{n}+\phi_{h}^{n-1}}{2 \Delta t}, \phi_{h}^{n+1}\right) \\
& -\left(\frac{3 \phi_{h}^{n+1}-4 \phi_{h}^{n}+\phi_{h}^{n-1}}{2 \Delta t}, \psi_{h}^{n+1}-\phi_{h}^{n+1}\right)-\left(\frac{3 \psi_{h}^{n+1}-3 \phi_{h}^{n+1}}{2 \Delta t}, \psi_{h}^{n+1}\right) \\
& +b\left(2 u^{n}-u^{n-1}, u^{n+1}, \psi_{h}^{n+1}\right)-b\left(2 u_{h}^{n}-u_{h}^{n-1}, \hat{u}_{h}^{n+1}, \psi_{h}^{n+1}\right) \\
& +\nu\left(\nabla \eta^{n+1}, \nabla \psi_{h}^{n+1}\right)-\nu\left\|\nabla \psi_{h}^{n+1}\right\|^{2}-\left(p^{n+1}-q_{h}, \nabla \cdot \psi_{h}^{n+1}\right)=\tau^{n+1}\left(\psi_{h}^{n+1}\right) .
\end{aligned}
$$

Here, $q_{h} \in Q_{h}$ is arbitrary. Furthermore, setting $v_{h}=\frac{3 \phi_{h}^{n+1}-4 \phi_{h}^{n}+\phi_{h}^{n-1}}{3} \in V_{h}$ in 4.7 and rearranging terms yields

$$
\begin{aligned}
& \left(\frac{3 \phi_{h}^{n+1}-4 \phi_{h}^{n}+\phi_{h}^{n-1}}{2 \Delta t}, \psi_{h}^{n+1}-\phi_{h}^{n+1}\right) \\
& =\frac{\gamma}{3}\left(\nabla \cdot\left(3 \phi_{h}^{n+1}-4 \phi_{h}^{n}+\phi_{h}^{n-1}\right), \nabla \cdot \phi_{h}^{n+1}\right)+\frac{\beta}{6 \Delta t}\left\|\nabla \cdot\left(3 \phi_{h}^{n+1}-4 \phi_{h}^{n}+\phi_{h}^{n-1}\right)\right\|^{2} \\
& \quad-\frac{\gamma}{3}\left(\nabla \cdot\left(3 \phi_{h}^{n+1}-4 \phi_{h}^{n}+\phi_{h}^{n-1}\right), \nabla \cdot \eta^{n+1}\right) \\
& \quad-\frac{\beta}{6 \Delta t}\left(\nabla \cdot\left(3 \eta^{n+1}-4 \eta^{n}+\eta^{n-1}\right), \nabla \cdot\left(3 \phi_{h}^{n+1}-4 \phi_{h}^{n}+\phi_{h}^{n-1}\right)\right) .
\end{aligned}
$$

Combine 4.18 and 4.19 and rearrange. Then,

$$
\begin{aligned}
& \left(\frac{3 \phi_{h}^{n+1}-4 \phi_{h}^{n}+\phi_{h}^{n-1}}{2 \Delta t}, \phi_{h}^{n+1}\right)+\frac{\gamma}{3}\left(\nabla \cdot\left(3 \phi_{h}^{n+1}-4 \phi_{h}^{n}+\phi_{h}^{n-1}\right), \nabla \cdot \phi^{n+1}\right) \\
& \quad+\frac{\beta}{6 \Delta t}\left\|\nabla \cdot\left(3 \phi_{h}^{n+1}-4 \phi_{h}^{n}+\phi_{h}^{n-1}\right)\right\|^{2}+\nu\left\|\nabla \psi_{h}^{n+1}\right\|^{2} \\
& \quad+\frac{3}{4 \Delta t}\left(\left\|\psi_{h}^{n+1}\right\|^{2}-\left\|\phi_{h}^{n+1}\right\|^{2}+\left\|\psi_{h}^{n+1}-\phi_{h}^{n+1}\right\|^{2}\right) \\
& =\left(\frac{3 \eta^{n+1}-4 \eta^{n}+\eta^{n-1}}{2 \Delta t}, \psi_{h}^{n+1}\right)+\frac{\gamma}{3}\left(\nabla \cdot\left(3 \phi_{h}^{n+1}-4 \phi_{h}^{n}+\phi_{h}^{n-1}\right), \nabla \cdot \eta^{n+1}\right) \\
& \quad+\frac{\beta}{6 \Delta t}\left(\nabla \cdot\left(3 \eta^{n+1}-4 \eta^{n}+\eta^{n-1}\right), \nabla \cdot\left(3 \phi_{h}^{n+1}-4 \phi_{h}^{n}+\phi_{h}^{n-1}\right)\right) \\
& \quad+b\left(2 u^{n}-u^{n-1}, u^{n+1}, \psi_{h}^{n+1}\right)-b\left(2 u_{h}^{n}-u_{h}^{n-1}, \hat{u}_{h}^{n+1}, \psi_{h}^{n+1}\right) \\
& \quad+\nu\left(\nabla \eta^{n+1}, \nabla \psi_{h}^{n+1}\right)-\left(p^{n+1}-q_{h}, \nabla \cdot \psi_{h}^{n+1}\right)-\tau^{n+1}\left(\psi_{h}^{n+1}\right) . \\
& \quad 9
\end{aligned}
$$


Multiplying (4.20) by $4 \Delta t$ and use 4.5. Then,

$$
\begin{aligned}
&\left\|\phi_{h}^{n+1}\right\|^{2}-\left\|\phi_{h}^{n}\right\|^{2}+\left\|2 \phi_{h}^{n+1}-\phi_{h}^{n}\right\|^{2}-\left\|2 \phi_{h}^{n}-\phi_{h}^{n-1}\right\|^{2}+\left\|\phi_{h}^{n+1}-2 \phi_{h}^{n}+\phi_{h}^{n-1}\right\|^{2} \\
& \quad+\left(\frac{2 \gamma \Delta t}{3}+\beta\right)\left(\left\|\nabla \cdot \phi_{h}^{n+1}\right\|^{2}-\left\|\nabla \cdot \phi_{h}^{n}\right\|^{2}+\left\|\nabla \cdot\left(2 \phi_{h}^{n+1}-\phi_{h}^{n}\right)\right\|^{2}\right. \\
&\left.\quad-\left\|\nabla \cdot\left(2 \phi_{h}^{n}-\phi_{h}^{n-1}\right)\right\|^{2}+\left\|\nabla \cdot\left(\phi_{h}^{n+1}-2 \phi_{h}^{n}+\phi_{h}^{n-1}\right)\right\|^{2}\right) \\
& \quad+\frac{2 \beta}{3}\left\|\nabla \cdot\left(3 \phi_{h}^{n+1}-4 \phi_{h}^{n}+\phi_{h}^{n-1}\right)\right\|^{2}+6\left\|\psi_{h}^{n+1}-\phi_{h}^{n+1}\right\|^{2} \\
& \quad+4 \nu \Delta t\left\|\nabla \psi_{h}^{n+1}\right\|^{2}+2 \gamma \Delta t\left\|\nabla \cdot \phi_{h}^{n+1}\right\|^{2} \\
& \leq 2\left(3 \eta^{n+1}-4 \eta^{n}+\eta^{n-1}, \psi_{h}^{n+1}\right)+\frac{4 \gamma \Delta t}{3}\left(\nabla \cdot\left(3 \phi_{h}^{n+1}-4 \phi_{h}^{n}+\phi_{h}^{n-1}\right), \nabla \cdot \eta^{n+1}\right) \\
&+\frac{2 \beta}{3}\left(\nabla \cdot\left(3 \eta^{n+1}-4 \eta^{n}+\eta^{n-1}\right), \nabla \cdot\left(3 \phi_{h}^{n+1}-4 \phi_{h}^{n}+\phi_{h}^{n-1}\right)\right) \\
&+4 \Delta t b\left(2 u^{n}-u^{n-1}, u^{n+1}, \psi_{h}^{n+1}\right)-4 \Delta t b\left(2 u_{h}^{n}-u_{h}^{n-1}, \hat{u}_{h}^{n+1}, \psi_{h}^{n+1}\right) \\
&+4 \nu \Delta t\left(\nabla \eta^{n+1}, \nabla \psi_{h}^{n+1}\right)-4 \Delta t\left(p^{n+1}-q_{h}, \nabla \cdot \psi_{h}^{n+1}\right)-4 \Delta t \tau^{n+1}\left(\psi_{h}^{n+1}\right) \\
&+C \beta d(1+2 \Delta t) \int_{t^{n-1}}^{t^{n+1}}\left\|\nabla \eta_{t}\right\|^{2} d t+2 \gamma d \Delta t\left\|\nabla \eta^{n+1}\right\|^{2} \\
&+\frac{\beta}{2}\left\|\nabla \cdot\left(\phi_{h}^{n+1}-2 \phi_{h}^{n+1}+\phi_{h}^{n}\right)\right\|^{2}+\beta \Delta t\left(\left\|\nabla \cdot\left(2 \phi_{h}^{n}-\phi_{h}^{n-1}\right)\right\|^{2}+\left\|\nabla \cdot \phi_{h}^{n}\right\|^{2}\right) .
\end{aligned}
$$

Next, we need to bound the terms on the right hand side of 4.21). Applying Lemma 2.2 . the Poincaré-Friedrichs inequality, and the Cauchy-Schwarz-Young inequality, for an arbitrary $\delta>0$, we have

$$
\begin{aligned}
& 2\left(3 \eta^{n+1}-4 \eta^{n}+\eta^{n-1}, \psi_{h}^{n+1}\right) \leq C\left\|3 \eta^{n+1}-4 \eta^{n}+\eta^{n-1}\right\|\left\|\nabla \psi_{h}^{n+1}\right\| \\
& \leq \frac{C}{\delta \nu} \int_{t^{n-1}}^{t^{n+1}}\left\|\eta_{t}\right\|^{2} d t+\delta \nu \Delta t\left\|\nabla \psi_{h}^{n+1}\right\|^{2} . \\
& \frac{4 \gamma \Delta t}{3}\left(\nabla \cdot\left(3 \phi_{h}^{n+1}-4 \phi_{h}^{n}+\phi_{h}^{n-1}\right), \nabla \cdot \eta^{n+1}\right) \\
& \leq \frac{4 \gamma \sqrt{d} \Delta t}{3}\left\|\nabla \cdot\left(3 \phi_{h}^{n+1}-4 \phi_{h}^{n}+\phi_{h}^{n-1}\right)\right\|\left\|\nabla \eta^{n+1}\right\| \\
& \leq \frac{\beta}{3}\left\|\nabla \cdot\left(3 \phi_{h}^{n+1}-4 \phi_{h}^{n}+\phi_{h}^{n-1}\right)\right\|^{2}+\frac{4 d \gamma^{2} \Delta t^{2}}{3 \beta}\left\|\nabla \eta^{n+1}\right\|^{2} . \\
& \frac{2 \beta}{3}\left(\nabla \cdot\left(3 \eta^{n+1}-4 \eta^{n}+\eta^{n-1}\right), \nabla \cdot\left(3 \phi_{h}^{n+1}-4 \phi_{h}^{n}+\phi_{h}^{n-1}\right)\right) \\
& \leq \frac{2 \beta \sqrt{d}}{3}\left\|\nabla\left(3 \eta^{n+1}-4 \eta^{n}+\eta^{n-1}\right)\right\|\left\|\nabla \cdot\left(3 \phi_{h}^{n+1}-4 \phi_{h}^{n}+\phi_{h}^{n-1}\right)\right\| \\
& \leq \frac{\beta}{3}\left\|\nabla \cdot\left(3 \phi_{h}^{n+1}-4 \phi_{h}^{n}+\phi_{h}^{n-1}\right)\right\|^{2}+\frac{C \beta d \Delta t}{3} \int_{t^{n-1}}^{t^{n+1}}\left\|\nabla \eta_{t}\right\|^{2} d t .
\end{aligned}
$$

Furthermore,

$$
\begin{gathered}
4 \nu \Delta t\left(\nabla \eta^{n+1}, \nabla \psi_{h}^{n+1}\right) \leq \frac{4 \nu \Delta t}{\delta}\left\|\nabla \eta^{n+1}\right\|^{2}+\delta \nu \Delta t\left\|\nabla \psi_{h}^{n+1}\right\|^{2} . \\
-4 \Delta t\left(p^{n+1}-q_{h}, \nabla \cdot \psi_{h}^{n+1}\right) \leq \frac{4 d \Delta t}{\delta \nu}\left\|p^{n+1}-q_{h}\right\|^{2}+\delta \nu \Delta t\left\|\nabla \psi_{h}^{n+1}\right\|^{2} . \\
10
\end{gathered}
$$


Applying Lemma 4.2 yields

$$
\begin{aligned}
& -4 \Delta t \tau^{n+1}\left(\psi_{h}^{n+1}\right) \\
& \leq \frac{C \Delta t^{4}}{\delta \nu} \int_{t^{n-1}}^{t^{n+1}}\left\|u_{t t t}\right\|_{-1}^{2} d t+\frac{C \Delta t^{4}}{\delta \nu} \int_{t^{n-1}}^{t^{n+1}}\left\|\nabla u_{t t}\right\|^{2} d t+\delta \nu \Delta t\left\|\nabla \psi_{h}^{n+1}\right\|^{2} .
\end{aligned}
$$

For the nonlinear terms, we treat them as follows. Adding and subtracting $4 \Delta t b\left(2 u_{h}^{n}-\right.$ $u_{h}^{n-1}, u^{n+1}, \psi_{h}^{n+1}$ ) yields

$$
\begin{aligned}
4 & \Delta t b\left(2 u^{n}-u^{n-1}, u^{n+1}, \psi_{h}^{n+1}\right)-4 \Delta t b\left(2 u_{h}^{n}-u_{h}^{n-1}, \hat{u}_{h}^{n+1}, \psi_{h}^{n+1}\right) \\
= & 4 \Delta t\left(b\left(2 \eta^{n}-\eta^{n-1}, u^{n+1}, \psi_{h}^{n+1}\right)-b\left(2 \phi_{h}^{n}-\phi_{h}^{n-1}, u^{n+1}, \psi_{h}^{n+1}\right)\right. \\
& \left.+b\left(2 \hat{u}_{h}^{n}-\hat{u}_{h}^{n-1}, \eta^{n+1}, \psi_{h}^{n+1}\right)\right) .
\end{aligned}
$$

Then,

$$
\begin{aligned}
& 4 \Delta t b\left(2 \eta^{n}-\eta^{n-1}, u^{n+1}, \psi_{h}^{n+1}\right) \leq 4 C \Delta t\left\|\nabla\left(2 \eta^{n}-\eta^{n-1}\right)\right\|\left\|\nabla u^{n+1}\right\|\left\|\nabla \psi_{h}^{n+1}\right\| \\
& \leq \frac{4 C \Delta t}{\delta \nu}\left\|\nabla\left(2 \eta^{n}-\eta^{n-1}\right)\right\|^{2}\left\|\nabla u^{n+1}\right\|^{2}+\delta \nu \Delta t\left\|\nabla \psi_{h}^{n+1}\right\|^{2} \\
& \leq \frac{16 C \Delta t}{\delta \nu}\left(\left\|\nabla \eta^{n}\right\|^{2}+\left\|\nabla \eta^{n-1}\right\|^{2}\right)\|\nabla u\|_{\infty, 0}^{2}+\delta \nu \Delta t\left\|\nabla \psi_{h}^{n+1}\right\|^{2}, \\
& -4 \Delta t b\left(2 \phi_{h}^{n}-\phi_{h}^{n-1}, u^{n+1}, \psi_{h}^{n+1}\right) \leq 4 C \Delta t\left\|2 \phi_{h}^{n}-\phi_{h}^{n-1}\right\|\left\|u^{n+1}\right\|_{2}\left\|\nabla \psi_{h}^{n+1}\right\| \\
& \leq \frac{4 C \Delta t}{\delta \nu}\left\|2 \phi_{h}^{n}-\phi_{h}^{n-1}\right\|^{2}\left\|u^{n+1}\right\|_{2}^{2}+\delta \nu \Delta t\left\|\nabla \psi_{h}^{n+1}\right\|^{2} \\
& \leq \frac{8 C \Delta t}{\delta \nu}\left(\left\|2 \phi_{h}^{n}-\phi_{h}^{n-1}\right\|^{2}+\left\|\phi_{h}^{n}\right\|^{2}\right)\left\|u^{n+1}\right\|_{2}^{2}+\delta \nu \Delta t\left\|\nabla \psi_{h}^{n+1}\right\|^{2}, \\
& 4 \Delta t b\left(2 \hat{u}_{h}^{n}-\hat{u}_{h}^{n-1}, \eta^{n+1}, \psi_{h}^{n+1}\right) \leq 4 C \Delta t\left\|\nabla\left(2 \hat{u}_{h}^{n}-\hat{u}_{h}^{n-1}\right)\right\|\left\|\nabla \eta^{n+1}\right\|\left\|\nabla \psi_{h}^{n+1}\right\| \\
& \leq \frac{4 C \Delta t}{\delta \nu}\left\|\nabla\left(2 \hat{u}_{h}^{n}-\hat{u}_{h}^{n-1}\right)\right\|^{2}\left\|\nabla \eta^{n+1}\right\|^{2}+\delta \nu \Delta t\left\|\nabla \psi_{h}^{n+1}\right\|^{2} \\
& \leq \frac{16 C \Delta t}{\delta \nu}\left(\left\|\nabla \hat{u}_{h}^{n}\right\|^{2}+\left\|\nabla \hat{u}_{h}^{n-1}\right\|^{2}\right)\|\nabla \eta\|_{\infty, 0}^{2}+\delta \nu \Delta t\left\|\nabla \psi_{h}^{n+1}\right\|^{2} .
\end{aligned}
$$

Setting $\delta=\frac{2}{7}$ and using the estimates 4.22 - 4.31 in 4.21 yields

$$
\begin{aligned}
& \left\|\phi_{h}^{n+1}\right\|^{2}-\left\|\phi_{h}^{n}\right\|^{2}+\left\|2 \phi_{h}^{n+1}-\phi_{h}^{n}\right\|^{2}-\left\|2 \phi_{h}^{n}-\phi_{h}^{n-1}\right\|^{2}+\left\|\phi_{h}^{n+1}-2 \phi_{h}^{n}+\phi_{h}^{n-1}\right\|^{2} \\
& \quad+\left(\frac{2 \gamma \Delta t}{3}+\beta\right)\left(\left\|\nabla \cdot \phi_{h}^{n+1}\right\|^{2}-\left\|\nabla \cdot \phi_{h}^{n}\right\|^{2}+\left\|\nabla \cdot\left(2 \phi_{h}^{n+1}-\phi_{h}^{n}\right)\right\|^{2}-\left\|\nabla \cdot\left(2 \phi_{h}^{n}-\phi_{h}^{n-1}\right)\right\|^{2}\right. \\
& \left.\quad+\frac{1}{2}\left\|\nabla \cdot\left(\phi_{h}^{n+1}-2 \phi_{h}^{n}+\phi_{h}^{n-1}\right)\right\|^{2}\right)+6\left\|\psi_{h}^{n+1}-\phi_{h}^{n+1}\right\|^{2}+2 \nu \Delta t\left\|\nabla \psi_{h}^{n+1}\right\|^{2}+2 \gamma \Delta t\left\|\nabla \cdot \phi_{h}^{n+1}\right\|^{2} \\
& \leq \frac{C \Delta t}{\nu}\left\|u^{n+1}\right\|_{2}^{2}\left(\left\|2 \phi_{h}^{n}-\phi_{h}^{n-1}\right\|^{2}+\left\|\phi_{h}^{n}\right\|^{2}\right)+\beta \Delta t\left(\left\|\nabla \cdot\left(2 \phi_{h}^{n}-\phi_{h}^{n-1}\right)\right\|^{2}+\left\|\nabla \cdot \phi_{h}^{n}\right\|^{2}\right) \\
& \quad+C \beta d \Delta t \int_{t^{n-1}}^{t^{n+1}}\left\|\nabla \eta_{t}\right\|^{2} d t+\frac{C}{\nu} \int_{t^{n-1}}^{t^{n+1}}\left\|\eta_{t}\right\|^{2} d t+C\left(\frac{d \gamma^{2} \Delta t}{\beta}+\nu\right) \Delta t\left\|\nabla \eta^{n+1}\right\|^{2} \\
& \quad+\frac{C d \Delta t}{\nu}\left\|p^{n+1}-q_{h}\right\|^{2}+\frac{C \Delta t^{4}}{\nu} \int_{t^{n-1}}^{t^{n+1}}\left\|u_{t t t}\right\|_{-1}^{2} d t+\frac{C \Delta t^{4}}{\nu} \int_{t^{n-1}}^{t^{n+1}}\left\|\nabla u_{t t}\right\|^{2} d t \\
& \quad+\frac{C \Delta t}{\nu}\left(\left\|\nabla \eta^{n}\right\|^{2}+\left\|\nabla \eta^{n-1}\right\|^{2}\right)+\frac{C \Delta t}{\nu}\left(\left\|\nabla \hat{u}_{h}^{n}\right\|^{2}+\left\|\nabla \hat{u}_{h}^{n-1}\right\|^{2}\right)\|\nabla \eta\|_{\infty, 0}^{2} .
\end{aligned}
$$


Sum 4.32 from $n=1$ to $N-1$ to get

$$
\begin{aligned}
&\left\|\phi_{h}^{N}\right\|^{2}+\left\|2 \phi_{h}^{N}-\phi_{h}^{N-1}\right\|^{2}+\left(\frac{2 \gamma \Delta t}{3}+\beta\right)\left(\left\|\nabla \cdot \phi_{h}^{N}\right\|^{2}+\left\|\nabla \cdot\left(2 \phi_{h}^{N}-\phi_{h}^{N-1}\right)\right\|^{2}\right) \\
& \quad+\frac{1}{2} \sum_{n=1}^{N-1}\left\|\nabla \cdot\left(\phi_{h}^{n+1}-2 \phi_{h}^{n}+\phi_{h}^{n-1}\right)\right\|^{2}+2 \nu \Delta t \sum_{n=1}^{N-1}\left\|\nabla \psi_{h}^{n+1}\right\|^{2}+2 \gamma \Delta t \sum_{n=1}^{N-1}\left\|\nabla \cdot \phi_{h}^{n+1}\right\|^{2} \\
& \leq \Delta t \sum_{n=1}^{N-1}\left(C \nu^{-1}\left\|u^{n+1}\right\|_{2}^{2}\left(\left\|\phi_{h}^{n}\right\|^{2}+\left\|2 \phi_{h}^{n}-\phi_{h}^{n-1}\right\|^{2}\right)+\beta\left(\left\|\nabla \cdot \phi_{h}^{n}\right\|^{2}+\left\|\nabla \cdot\left(2 \phi_{h}^{n}-\phi_{h}^{n-1}\right)\right\|^{2}\right)\right) \\
&+\frac{C \beta d \Delta t}{3}\left\|\nabla \eta_{t}\right\|_{2,0}^{2}+\frac{C}{\nu}\left\|\eta_{t}\right\|_{2,0}^{2}+C\left(\frac{d \gamma^{2} \Delta t}{\beta}+\nu+\frac{1}{\nu}\right)\|\nabla \eta\|_{2,0}^{2} \\
&+\left.\frac{C d}{\nu}\left\|p-q_{h}\right\|\right|_{2,0} ^{2}+\frac{C \Delta t^{4}}{\nu}\left\|u_{t t t}\right\|_{2,-1}^{2}+\frac{C \Delta t^{4}}{\nu}\left\|\nabla u_{t t}\right\|_{2,0}^{2}+\frac{C}{\nu^{2}}\left(\nu \Delta t \sum_{n=0}^{N-1}\left\|\nabla \hat{u}_{h}^{n}\right\|^{2}\right)\|\nabla \eta\|_{\infty, 0}^{2} \\
&+\left\|\phi_{h}^{1}\right\|^{2}+\left\|2 \phi_{h}^{1}-\phi_{h}^{0}\right\|^{2}+\left(\frac{2 \gamma \Delta t}{3}+\beta\right)\left(\left\|\nabla \cdot \phi_{h}^{1}\right\|^{2}+\left\|\nabla \cdot\left(2 \phi_{h}^{1}-\phi_{h}^{0}\right)\right\|^{2}\right) .
\end{aligned}
$$

Denote $C^{*}=\max \left\{\frac{C}{\nu} \mid\|u\|_{2,2}^{2}, 1\right\}$. Then, Lemma 2.3 , the boundedness of $\nu \Delta t \sum_{n=1}^{N-1}\left\|\nabla \hat{u}_{h}^{n+1}\right\|^{2}$ (Theorem 3.3), and taking infimums over $V_{h}$ and $Q_{h}$ yield

$$
\begin{aligned}
\| \phi_{h}^{N} & \left\|^{2}+\right\| 2 \phi_{h}^{N}-\phi_{h}^{N-1} \|^{2}+\left(\frac{2 \gamma \Delta t}{3}+\beta\right)\left(\left\|\nabla \cdot \phi_{h}^{N}\right\|^{2}+\left\|\nabla \cdot\left(2 \phi_{h}^{N}-\phi_{h}^{N-1}\right)\right\|^{2}\right) \\
& +\frac{1}{2} \sum_{n=1}^{N-1}\left\|\nabla \cdot\left(\phi_{h}^{n+1}-2 \phi_{h}^{n}+\phi_{h}^{n-1}\right)\right\|^{2}+2 \nu \Delta t \sum_{n=1}^{N-1}\left\|\nabla \psi_{h}^{n+1}\right\|^{2}+2 \gamma \Delta t \sum_{n=1}^{N-1}\left\|\nabla \cdot \phi_{h}^{n+1}\right\|^{2} \\
\leq & C \exp \left(C^{*} T\right)\left\{\operatorname { i n f } _ { v _ { h } \in V _ { h } } \left(\beta(1+\Delta t)\left\|\nabla\left(u-v_{h}\right)_{t}\right\|_{2,0}^{2}+\frac{1}{\nu}\left\|\left(u-v_{h}\right)_{t}\right\|_{2,0}^{2}\right.\right. \\
& \left.+\left(\frac{\gamma^{2} \Delta t}{\beta}+\nu+\frac{1}{\nu}\right) \mid\left\|\nabla\left(u-v_{h}\right)\right\|_{2,0}^{2}+\frac{1}{\nu^{2}}\left\|\nabla\left(u-v_{h}\right)\right\|_{\infty, 0}^{2}\right) \\
& +\frac{1}{\nu} \inf _{q_{h} \in Q_{h}}\left|\left\|p-q_{h}\right\|\right|_{2,0}^{2}+\frac{1}{\nu} \Delta t^{4} \\
& \left.+\left\|\phi_{h}^{1}\right\|^{2}+\left\|2 \phi_{h}^{1}-\phi_{h}^{0}\right\|^{2}+\left(\frac{2 \gamma \Delta t}{3}+\beta\right)\left(\left\|\nabla \cdot \phi_{h}^{1}\right\|^{2}+\left\|\nabla \cdot\left(2 \phi_{h}^{1}-\phi_{h}^{0}\right)\right\|^{2}\right)\right\}
\end{aligned}
$$

Then, using Lemma 2.1 and the triangle inequality completes the proof. $\mathrm{c}$

The above result has dependence on $\beta^{-1}$. Consequently, we consider the convergency of BDF2-mgd when $\beta=0$ separately.

TheOREm 4.5. Assume the true solution $u, p$ satisfy (4.2) and (4.12). Then, when $\beta=0$, we have the following estimates for BDF2-mgd.

$$
\begin{aligned}
& \left\|e_{u}^{N}\right\|^{2}+\left\|2 e_{u}^{N}-e_{u}^{N-1}\right\|^{2}+\frac{2 \gamma \Delta t}{3}\left(\left\|\nabla \cdot e_{u}^{N}\right\|^{2}+\left\|\nabla \cdot\left(2 e_{u}^{N}-e_{u}^{N-1}\right)\right\|^{2}\right) \\
& +2 \nu \Delta t \sum_{n=1}^{N-1}\left\|\nabla e_{\hat{u}}^{n+1}\right\|^{2}+2 \gamma \Delta t \sum_{n=1}^{N-1}\left\|\nabla \cdot e_{u}^{n+1}\right\|^{2} \\
& \leq C \exp \left(C^{* *} T\right)\left\{\operatorname { i n f } _ { v _ { h } \in X _ { h } } \left(\frac{1}{\nu}\left\|\left(u-v_{h}\right)_{t}\right\|_{2,0}^{2}+\left\|u-v_{h}\right\|_{\infty, 0}^{2}+\left.\left(\gamma+\nu+\frac{1}{\nu}\right)\left\|\nabla\left(u-v_{h}\right)\right\|\right|_{2,0} ^{\not 4}\right.\right. \\
& \left.+\left(\frac{2 \gamma \Delta t}{3}+\frac{1}{\nu^{2}}\right)\left\|\nabla\left(u-v_{h}\right)\right\|_{\infty, 0}^{2}\right)+\frac{1}{\nu} \inf _{q_{h} \in Q_{h}} \mid\left\|p-q_{h}\right\|_{2,0}^{2}+\frac{1}{\nu} \Delta t^{4} \\
& \left.+\left\|e_{u}^{1}\right\|^{2}+\left\|2 e_{u}^{1}-e_{u}^{0}\right\|^{2}+\gamma \Delta t\left\|\nabla \cdot e_{u}^{1}\right\|^{2}+\gamma \Delta t\left\|\nabla \cdot\left(2 e_{u}^{1}-e_{u}^{0}\right)\right\|^{2}\right\} .
\end{aligned}
$$


Proof. Similar to 4.21, we have

$$
\begin{aligned}
& \left\|\phi_{h}^{n+1}\right\|^{2}-\left\|\phi_{h}^{n}\right\|^{2}+\left\|2 \phi_{h}^{n+1}-\phi_{h}^{n}\right\|^{2}-\left\|2 \phi_{h}^{n}-\phi_{h}^{n-1}\right\|^{2}+\left\|\phi_{h}^{n+1}-2 \phi_{h}^{n}+\phi_{h}^{n-1}\right\|^{2} \\
& \quad+\frac{2 \gamma \Delta t}{3}\left(\left\|\nabla \cdot \phi_{h}^{n+1}\right\|^{2}-\left\|\nabla \cdot \phi_{h}^{n}\right\|^{2}+\left\|\nabla \cdot\left(2 \phi_{h}^{n+1}-\phi_{h}^{n}\right)\right\|^{2}\right. \\
& \left.\quad-\left\|\nabla \cdot\left(2 \phi_{h}^{n}-\phi_{h}^{n-1}\right)\right\|^{2}+\left\|\nabla \cdot\left(\phi_{h}^{n+1}-2 \phi_{h}^{n}+\phi_{h}^{n-1}\right)\right\|^{2}\right) \\
& \quad+6\left\|\psi_{h}^{n+1}-\phi_{h}^{n+1}\right\|^{2}+4 \nu \Delta t\left\|\nabla \psi_{h}^{n+1}\right\|^{2}+2 \gamma \Delta t\left\|\nabla \cdot \phi_{h}^{n+1}\right\|^{2} \\
& \leq \\
& 2\left(3 \eta^{n+1}-4 \eta^{n}+\eta^{n-1}, \psi_{h}^{n+1}\right)+\frac{4 \gamma \Delta t}{3}\left(\nabla \cdot\left(3 \phi_{h}^{n+1}-4 \phi_{h}^{n}+\phi_{h}^{n-1}\right), \nabla \cdot \eta^{n+1}\right) \\
& \quad+4 \Delta t b\left(2 u^{n}-u^{n-1}, u^{n+1}, \psi_{h}^{n+1}\right)-4 \Delta t b\left(2 u_{h}^{n}-u_{h}^{n-1}, \hat{u}_{h}^{n+1}, \psi_{h}^{n+1}\right) \\
& \quad+4 \nu \Delta t\left(\nabla \eta^{n+1}, \nabla \psi_{h}^{n+1}\right)-4 \Delta t\left(p^{n+1}-q_{h}, \nabla \cdot \psi_{h}^{n+1}\right)-4 \Delta t \tau^{n+1}\left(\psi_{h}^{n+1}\right) \\
& \quad+\frac{4 \gamma d \Delta t}{3}\left\|\nabla \eta^{n+1}\right\|^{2} .
\end{aligned}
$$

Since $\beta=0$, we estimate $\frac{4 \gamma \Delta t}{3}\left(\nabla \cdot\left(3 \phi_{h}^{n+1}-4 \phi_{h}^{n}+\phi_{h}^{n-1}\right), \nabla \cdot \eta^{n+1}\right)$ as follows,

$$
\begin{aligned}
& \frac{4 \gamma \Delta t}{3}\left(\nabla \cdot\left(3 \phi_{h}^{n+1}-4 \phi_{h}^{n}+\phi_{h}^{n-1}\right), \nabla \cdot \eta^{n+1}\right) \\
& \leq \frac{4 \gamma \sqrt{d} \Delta t}{3}\left(2\left\|\nabla \cdot \phi_{h}^{n+1}\right\|+2\left\|\nabla \cdot \phi_{h}^{n}\right\|+\left\|\nabla \cdot\left(\phi_{h}^{n+1}-2 \phi_{h}^{n}+\phi_{h}^{n-1}\right)\right\|\right)\left\|\nabla \eta^{n+1}\right\| \\
& \leq \frac{2 \gamma \Delta t}{3}\left\|\nabla \cdot\left(\phi_{h}^{n+1}-2 \phi_{h}^{n}+\phi_{h}^{n-1}\right)\right\|^{2}+\frac{\gamma \Delta t}{2}\left\|\nabla \cdot \phi_{h}^{n+1}\right\|^{2}+\frac{\gamma \Delta t}{2}\left\|\nabla \cdot \phi_{h}^{n}\right\|^{2}+\frac{70 \gamma d \Delta t}{9}\left\|\nabla \eta^{n+1}\right\|^{2} .
\end{aligned}
$$

Then we have

$$
\begin{aligned}
& \left\|\phi_{h}^{n+1}\right\|^{2}-\left\|\phi_{h}^{n}\right\|^{2}+\left\|2 \phi_{h}^{n+1}-\phi_{h}^{n}\right\|^{2}-\left\|2 \phi_{h}^{n}-\phi_{h}^{n-1}\right\|^{2}+\left\|\phi_{h}^{n+1}-2 \phi_{h}^{n}+\phi_{h}^{n-1}\right\|^{2} \\
& \quad+\frac{2 \gamma \Delta t}{3}\left(\left\|\nabla \cdot \phi_{h}^{n+1}\right\|^{2}-\left\|\nabla \cdot \phi_{h}^{n}\right\|^{2}+\left\|\nabla \cdot\left(2 \phi_{h}^{n+1}-\phi_{h}^{n}\right)\right\|^{2}-\left\|\nabla \cdot\left(2 \phi_{h}^{n}-\phi_{h}^{n-1}\right)\right\|^{2}\right) \\
& \quad+\frac{\gamma \Delta t}{2}\left(\left\|\nabla \cdot \phi_{h}^{n+1}\right\|^{2}-\left\|\nabla \cdot \phi_{h}^{n}\right\|^{2}\right)+6\left\|\psi_{h}^{n+1}-\phi_{h}^{n+1}\right\|^{2}+2 \nu \Delta t\left\|\nabla \psi_{h}^{n+1}\right\|^{2}+\gamma \Delta t\left\|\nabla \cdot \phi_{h}^{n+1}\right\|^{2} \\
& \leq \\
& \quad \frac{C \Delta t}{\nu}\left\|u^{n+1}\right\|_{2}^{2}\left(\left\|2 \phi_{h}^{n}-\phi_{h}^{n-1}\right\|^{2}+\left\|\phi_{h}^{n}\right\|^{2}+\left\|\phi_{h}^{n-1}\right\|^{2}\right) \\
& \quad+\frac{C}{\nu} \int_{t^{n-1}}^{t^{n+1}}\left\|\eta_{t}\right\|^{2} d t+C(\nu+\gamma d) \Delta t\left\|\nabla \eta^{n+1}\right\|^{2} \\
& \quad+\frac{C d \Delta t}{\nu}\left\|p^{n+1}-q_{h}\right\|^{2}+\frac{C \Delta t^{4}}{\nu} \int_{t^{n-1}}^{t^{n+1}}\left\|u_{t t t}\right\|_{-1}^{2} d t+\frac{C \Delta t^{4}}{\nu} \int_{t^{n-1}}^{t^{n+1}}\left\|\nabla u_{t t}\right\|^{2} d t \\
& \quad+\frac{C \Delta t}{\nu}\left(\left\|\nabla \eta^{n}\right\|^{2}+\left\|\nabla \eta^{n-1}\right\|^{2}\right)+\frac{C \Delta t}{\nu}\left(\left\|\nabla \hat{u}_{h}^{n}\right\|^{2}+\left\|\nabla \hat{u}_{h}^{n-1}\right\|^{2}\right)\|\nabla \eta\|_{\infty, 0}^{2} .
\end{aligned}
$$


Sum (4.38) from $n=1$ to $N-1$ to get

$$
\begin{aligned}
& \left\|\phi_{h}^{N}\right\|^{2}+\left\|2 \phi_{h}^{N}-\phi_{h}^{N-1}\right\|^{2}+\frac{2 \gamma \Delta t}{3}\left(\left\|\nabla \cdot \phi_{h}^{N}\right\|^{2}+\left\|\nabla \cdot\left(2 \phi_{h}^{N}-\phi_{h}^{N-1}\right)\right\|^{2}\right) \\
& \quad+2 \nu \Delta t \sum_{n=1}^{N-1}\left\|\nabla \psi_{h}^{n+1}\right\|^{2}+\gamma \Delta t \sum_{n=1}^{N-1}\left\|\nabla \cdot \phi_{h}^{n+1}\right\|^{2} \\
& \leq \frac{C \Delta t}{\nu} \sum_{n=1}^{N-1}\left\|u^{n+1}\right\|_{2}^{2}\left(\left\|\phi_{h}^{n}\right\|^{2}+\left\|2 \phi_{h}^{n}-\phi_{h}^{n-1}\right\|^{2}\right)+C\left(\gamma d+\nu+\frac{1}{\nu}\right) \mid\|\nabla \eta\|_{2,0}^{2} \\
& \quad+\frac{C}{\nu}\left\|\eta_{t}\right\|_{2,0}^{2}+\frac{C d}{\nu} \mid\left\|p-q_{h}\right\|_{2,0}^{2}+\frac{C \Delta t^{4}}{\nu}\left\|u_{t t t}\right\|_{2,-1}^{2}+\frac{C \Delta t^{4}}{\nu}\left\|\nabla u_{t t}\right\|_{2,0}^{2}+\frac{C}{\nu^{2}}\|\nabla \eta\|_{\infty, 0}^{2} \\
& \left.\quad+\left\|\phi_{h}^{1}\right\|^{2}+\left\|2 \phi_{h}^{1}-\phi_{h}^{0}\right\|^{2}+\frac{7 \gamma \Delta t}{6}\left\|\nabla \cdot \phi_{h}^{1}\right\|^{2}+\frac{2 \gamma \Delta t}{3}\left\|\nabla \cdot\left(2 \phi_{h}^{1}-\phi_{h}^{0}\right)\right\|^{2}\right) .
\end{aligned}
$$

Denote $C^{* *}=\frac{C}{\nu} \mid\|u\|_{2,2}^{2}$. The result then follows by similar arguments as in Theorem 4.4 .4

Corollary 4.6. Under the assumptions of Theorem 4.4, suppose that $\left(X_{h}, Q_{h}\right)$ is given by P2-P1 Taylor-Hood approximation elements $(k=2, m=1)$. Then, the following estimate holds for BDF2-mgd.

$$
\begin{aligned}
& \left\|e_{u}^{N}\right\|^{2}+\left\|2 e_{u}^{N}-e_{u}^{N-1}\right\|^{2}+\left(\frac{2 \gamma \Delta t}{3}+\beta\right)\left(\left\|\nabla \cdot e_{u}^{N}\right\|^{2}+\frac{1}{2}\left\|\nabla \cdot\left(2 e_{u}^{N}-e_{u}^{N-1}\right)\right\|^{2}\right) \\
& \quad+2 \nu \Delta t \sum_{n=1}^{N-1}\left\|\nabla e_{\hat{u}}^{n+1}\right\|^{2}+2 \gamma \Delta t \sum_{n=1}^{N-1}\left\|\nabla \cdot e_{u}^{n+1}\right\|^{2} \\
& \leq C\left(h^{6}+h^{4}+\Delta t h^{4}+\Delta t^{4}\right. \\
& \left.\quad+\left\|e_{u}^{1}\right\|^{2}+\left\|2 e_{u}^{1}-e_{u}^{0}\right\|^{2}+(\gamma \Delta t+\beta)\left(\left\|\nabla \cdot e_{u}^{1}\right\|^{2}+\left\|\nabla \cdot\left(2 e_{u}^{1}-e_{u}^{0}\right)\right\|^{2}\right)\right) .
\end{aligned}
$$

5. Numerical Tests. In this section, we consider three test problems to illustrate the stability, convergence, and effectiveness of BDF2-mgd. First, we consider the Taylor-Green benchmark problem to compute convergence rates and test both computational efficiency and pressure-robustness. We follow with 2D channel flow over a step, where the effect of BDF2-mgd on reducing the divergence error is illustrated. Moreover, it is shown how $\gamma$ and $\beta$ influence this effect. Finally, we simulate flow past a cylinder to further present the effectiveness of BDF2-mgd. For all tests, we compare BDF2-mgd with BDF2 (Non-Stabilized) and BDF2 with standard grad-div stabilization (Standard Stabilized). All tests are implemented using FreeFem $++[32$.

5.1. Test of Convergence and Pressure Robustness. The Taylor-Green benchmark problem is commonly used to test convergence rates of new algorithms. As such, we first illustrate convergence rates. The domain is $[0,1] \times[0,1]$ and final time is $T=1$. Finite element meshes are generated via Delaunay-Vornoi triangulations with $m$ points on each side of the boundary. The true solution is given by

$$
\begin{aligned}
& u(x, y, t)=(-\cos (\omega \pi x) \sin (\omega \pi y), \sin (\omega \pi x) \cos (\omega \pi y)) \exp \left(-2 \omega^{2} \pi^{2} t / \tau\right), \\
& p(x, y, t)=-\frac{1}{4}(\cos (2 \omega \pi x)+\cos (2 \omega \pi y)) \exp \left(-4 \omega^{2} \pi^{2} t / \tau\right) .
\end{aligned}
$$

Here, $\omega=1, \tau=100$, and $R e=\frac{1}{\nu}=100$. The body force $f$, initial condition, and boundary condition are determined by the true solution. The grad-div parameters are set to $\gamma=1$, $\beta=0.2$. The time step is $\Delta t=1 / m$ where we vary $m=16,24,32,40$, and 48 to calculate 


\begin{tabular}{ccccccccc}
\hline$m$ & $\left|\left\|e_{u}\right\|\right|_{\infty, 0}$ & Rate & $\mid\left\|\nabla \cdot e_{u}\right\|_{\infty, 0}$ & Rate & $\left|\left\|\nabla \cdot e_{u}\right\|\right|_{2,0}$ & Rate & $\left.\left\|e_{p}\right\|\right|_{2,0}$ & Rate \\
\hline 16 & $2.47 \mathrm{E}-04$ & - & $3.33 \mathrm{E}-03$ & - & $2.82 \mathrm{E}-03$ & - & $1.26 \mathrm{E}-04$ & - \\
24 & $8.07 \mathrm{E}-05$ & 2.76 & $1.37 \mathrm{E}-03$ & 2.19 & $1.18 \mathrm{E}-03$ & 2.15 & $6.28 \mathrm{E}-05$ & 1.72 \\
32 & $3.54 \mathrm{E}-05$ & 2.86 & $7.21 \mathrm{E}-04$ & 2.24 & $6.24 \mathrm{E}-04$ & 2.21 & $2.87 \mathrm{E}-05$ & 2.73 \\
40 & $1.90 \mathrm{E}-05$ & 2.79 & $5.00 \mathrm{E}-04$ & 1.64 & $4.34 \mathrm{E}-04$ & 1.63 & $2.08 \mathrm{E}-05$ & 1.43 \\
48 & $1.12 \mathrm{E}-05$ & 2.88 & $3.58 \mathrm{E}-04$ & 1.83 & $3.11 \mathrm{E}-04$ & 1.82 & $1.32 \mathrm{E}-05$ & 2.50 \\
\hline
\end{tabular}

Errors and rates of velocity and pressure for BDF2-mgd using the Taylor-Hood element.

convergence rates. Table 5.1 presents the results which are consistent with our theoretical analysis.

To test computational efficiency, we set $m=32$ and vary $\gamma$ and $\beta$. We compare computational times of Standard Stabilized and BDF2-mgd; for $\gamma=\beta=0$, Standard Stabilized is equivalent to Non-Stabilized. For Standard Stabilized and Step 1 of BDF2-mgd, we use a standard GMRES solver. If GMRES fails to converge at a single iterate, we denote the result with an "F". For Step 2 of BDF2-mgd, since it leads to an SPD system with same sparse coefficient matrix, at each timestep, we use UMFPACK. The results are presented in Table 5.2 . The computing time of Standard Stabilized generally increases as $\gamma$ and $\beta$ increase. However, computing time of BDF2-mgd is unaffected and therefore increasingly more efficient than Standard Stabilized. Interestingly, GMRES fails to converge when $\gamma \simeq 20$ and $\beta \simeq 0.8$, which are not very large values.

Lastly, we consider the issue of pressure-robustness. An advantage of grad-div stabilization is that appropriate selection of the grad-div parameter $\gamma$ can reduce the effect of the pressure error on the velocity error. Generally, for non-stabilized methods, velocity error estimates result in $\nu^{-1} \inf _{q_{h} \in Q_{h}}\left|\left\|p-p_{h}\right\|\right|_{2,0}^{2}$ on the right hand side; see, e.g., Theorem 24 on p. 168 of [17]. This same term appears for BDF2-mgd in Theorems 4.4 and 4.5 . However, for standard grad-div stabilized methods, this term is replaced by $\gamma^{-1} \inf _{q_{h} \in Q_{h}}\left|\left\|p-p_{h}\right\|\right|_{2,0}^{2}$ in theoretical analyses. To investigate the sharpness of our results, we vary $R e$ while fixing $\Delta t=1 / m=1 / 32$ and $\gamma=1$, $\beta=0.2$. We compare the velocity and pressure errors of Non-Stabilized, Standard Stabilized, and BDF2-mgd. Results are presented in Table 5.3. It is clear that velocity errors of NonStabilized, especially for the divergence and gradient, grow as Re increases; this is consistent with the corresponding theoretical result. Alternatively, as $R e$ is increased, velocity errors of Standard Stabilized and BDF2-mgd are consistent with one another and maintain good approximations. This suggests that the effect of Re appearing in our analysis is not sharp. This is an open problem, Section 6 .

\begin{tabular}{|c|c|c|c|c|c|c|c|c|c|c|}
\hline Parameter & & $\iota_{h}-u \|\left.\right|_{\infty, 0}$ & & & $\left(u_{h}-u\right) \|\left.\right|_{2}$ & & & $\left(u_{h}-u\right) \|\left.\right|_{2,}$ & & $\left\|p_{h}-p\right\| \|_{2, \mathrm{c}}$ \\
\hline $\operatorname{Re}$ & Non-Stabilized & $\begin{array}{c}\text { Standard } \\
\text { Stabilized }\end{array}$ & BDF2-mgd & Non-Stabilized & $\begin{array}{l}\text { Standard } \\
\text { Stabilized }\end{array}$ & BDF2-mgd & Non-Stabilized & $\begin{array}{l}\text { Standard } \\
\text { Stabilized }\end{array}$ & BDF2-mgd & All \\
\hline 1 & $1.26 \mathrm{E}-03$ & $1.26 \mathrm{E}-03$ & $1.26 \mathrm{E}-03$ & $3.61 \mathrm{E}-05$ & $2.43 \mathrm{E}-05$ & $5.33 \mathrm{E}-05$ & $4.05 \mathrm{E}-03$ & $4.05 \mathrm{E}-03$ & $4.09 \mathrm{E}-03$ & $5.79 \mathrm{E}-07$ \\
\hline $1 \mathrm{e} 1$ & $2.45 \mathrm{E}-05$ & $2.42 \mathrm{E}-05$ & $2.70 \mathrm{E}-05$ & 4.71E-04 & $1.39 \mathrm{E}-04$ & $2.01 \mathrm{E}-04$ & $5.48 \mathrm{E}-04$ & $3.67 \mathrm{E}-04$ & $2.07 \mathrm{E}-03$ & $1.09 \mathrm{E}-05$ \\
\hline $1 \mathrm{e} 2$ & $9.95 \mathrm{E}-05$ & $1.80 \mathrm{E}-05$ & $3.57 \mathrm{E}-05$ & $1.20 \mathrm{E}-02$ & $5.40 \mathrm{E}-04$ & 6.44E-04 & $1.27 \mathrm{E}-02$ & $2.80 \mathrm{E}-03$ & $6.65 \mathrm{E}-03$ & $2.99 \mathrm{E}-05$ \\
\hline $1 \mathrm{e} 3$ & $1.05 \mathrm{E}-03$ & $5.85 \mathrm{E}-05$ & $8.90 \mathrm{E}-05$ & $1.03 \mathrm{E}-01$ & $7.20 \mathrm{E}-04$ & $7.51 \mathrm{E}-04$ & $1.31 \mathrm{E}-01$ & $9.04 \mathrm{E}-03$ & $1.15 \mathrm{E}-02$ & $3.57 \mathrm{E}-05$ \\
\hline $1 \mathrm{e} 4$ & $1.30 \mathrm{E}-01$ & $2.23 \mathrm{E}-04$ & $2.62 \mathrm{E}-04$ & 6.27 & $7.61 \mathrm{E}-04$ & $7.78 \mathrm{E}-04$ & 7.81 & $2.40 \mathrm{E}-02$ & $3.01 \mathrm{E}-02$ & $3.64 \mathrm{E}-05$ \\
\hline $1 \mathrm{e} 5$ & $3.57 \mathrm{E}-01$ & $3.99 \mathrm{E}-04$ & $3.50 \mathrm{E}-04$ & 16.36 & $7.76 \mathrm{E}-04$ & $7.84 \mathrm{E}-04$ & 25.23 & $3.61 \mathrm{E}-02$ & $3.95 \mathrm{E}-02$ & $3.64 \mathrm{E}-05$ \\
\hline $1 \mathrm{e} 6$ & 4.33E-01 & $4.32 \mathrm{E}-04$ & 3.63E-04 & 20.34 & $7.78 \mathrm{E}-04$ & $7.85 \mathrm{E}-04$ & 32.44 & $3.84 \mathrm{E}-02$ & $4.09 \mathrm{E}-02$ & $3.64 \mathrm{E}-05$ \\
\hline
\end{tabular}

Comparison of velocity and pressure errors with increasing Re.

5.2. 2D Channel Flow Over a Step. We now illustrate the effect of Step 2 of BDF2-mgd by comparing Non-Stabilized, Standard Stabilized, and BDF2-mgd simulations of $2 \mathrm{D}$ channel flow over a step [6, 14]. The channel considered here is $[0,40] \times[0,10]$ with a $1 \times 1$ step on the bottom for $x \in[5,6]$. A flow with $\nu=1 / 600$ passes though this channel from left to right. For 


\begin{tabular}{cccc}
\hline \multicolumn{2}{c}{ Parameters } & \multicolumn{2}{c}{ Time $(\mathrm{s})$} \\
$\beta$ & $\gamma$ & Standard Stabilized & BDF2-mgd \\
\hline 0 & 0 & $\mathbf{1 7 . 7 7}$ & $\mathbf{2 5 . 0 9}$ \\
0 & 0.2 & $\mathbf{3 0 . 9 1}$ & $\mathbf{2 0 . 1 0}$ \\
0 & 2 & $\mathbf{5 5 . 2 9}$ & $\mathbf{2 0 . 4 5}$ \\
0 & 20 & $\mathrm{~F}(\mathbf{3 3 9 . 0 1})$ & $\mathbf{2 7 . 9 9}$ \\
0 & 200 & $\mathrm{~F}(\mathbf{5 0 7 . 4 1})$ & $\mathbf{2 3 . 8 8}$ \\
0 & 2,000 & $\mathrm{~F}(\mathbf{4 2 1 . 6 6})$ & $\mathbf{1 7 . 3 4}$ \\
0 & 20,000 & $\mathrm{~F}(\mathbf{2 7 . 4 4})$ & $\mathbf{2 0 . 0 4}$ \\
0.01 & 0.2 & 27.79 & 22.28 \\
0.02 & 0.2 & 32.89 & 22.33 \\
0.04 & 0.2 & 64.37 & 21.68 \\
0.08 & 0.2 & 69.31 & 23.97 \\
0.8 & 0.2 & $\mathrm{~F}$ & 25.87 \\
8 & 0.2 & $\mathrm{~F}$ & 19.53 \\
80 & 0.2 & $\mathrm{~F}$ & 21.20 \\
800 & 0.2 & $\mathrm{~F}$ & 17.43 \\
8,000 & 0.2 & $\mathrm{~F}$ & 18.64 \\
\hline
\end{tabular}

Computational time and solver breakdown for Standard and BDF2-mgd with increasing grad-div parameters.

boundary conditions, the left inlet and right outlet are given by

$$
\begin{aligned}
& u(0, y, t)=u(40, y, t)=y(10-y) / 25, \\
& v(0, y, t)=v(40, y, t)=0 .
\end{aligned}
$$

No-slip, $u=0$, boundary conditions are imposed elsewhere. Taylor-Hood elements are used, comprising a mesh with 31,089 degrees of freedom. The body force $f=0$, final time $T=40$, and time step $\Delta t=0.01$. The selected grad-div parameters are $\gamma=0.1,0.2,1$ and $\beta=0,0.1,0.2,1$. $\left\|\nabla \cdot u\left(t^{n}\right)\right\|$ is computed and plotted in Figure 5.1. Also, plots of flow speed and divergence contours, at the final time, with $\gamma=1, \beta=0$, are presented in Figure 5.2 .

As shown in Figure 5.1. Step 2 of BDF2-mgd greatly reduces the divergence error $\|\nabla \cdot u\|$ compared with Non-Stabilized. Observing the curves of different $\gamma$ and $\beta$, it's interesting to find that the value of $\beta$ determines the minimum divergence error that can be reached in the beginning and the value of $\gamma$ determines the long-time divergence error. This is consistent with [5. In Figure 5.2, we see that results for Step 2 of BDF2-mgd are consistent with Standard Stablilzed; both reduce divergence error, especially around the step.

5.3. 2D Channel Flow Past a Cylinder. In order to further test the effectiveness of BDF2-mgd, we consider channel flow past a cylinder [31. Like the Taylor-Green benchmark, this is a common test problem for new algorithms. The channel domain is $[0,2.2] \times[0,0.41]$ with a cylinder of diameter 0.1 within. The center of the cylinder is $(0.2,0.2)$. A flow with $\nu=0.001, \rho=1$ passes though this channel from left to right. No body forces are present, $f=0$. Left in-flow and right out-flow boundaries are given by

$$
\begin{aligned}
& u(0, y, t)=u(2.2, y, t)=\frac{6 y(0.41-y)}{0.41^{2}} \sin \left(\frac{\pi t}{8}\right), \\
& v(0, y, t)=v(2.2, y, t)=0 .
\end{aligned}
$$

The no-slip boundary condition is prescribed elsewhere.

We use Taylor-Hood elements on a mesh with 41,042 degree of freedom and final time $T=8$. The time step is $\Delta t=0.001$. The grad-div parameters are set to $\gamma=5 \nu$ and 


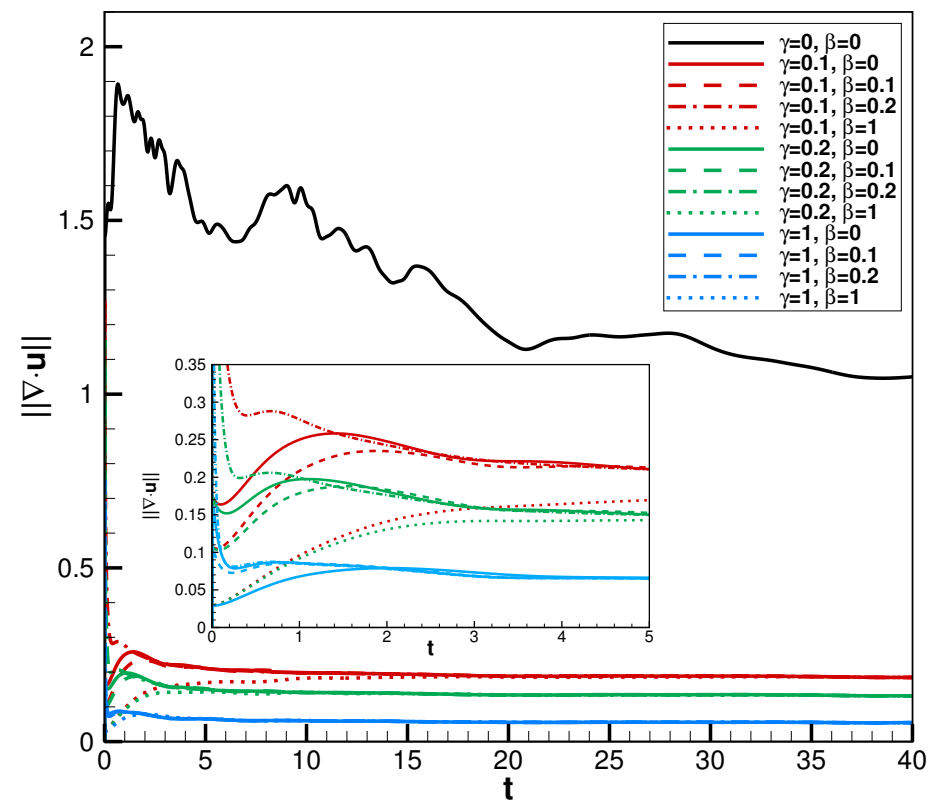

FIG. 5.1. $\|\nabla \cdot u\|$ vs time for Non-Stabilized and BDF2-mgd.
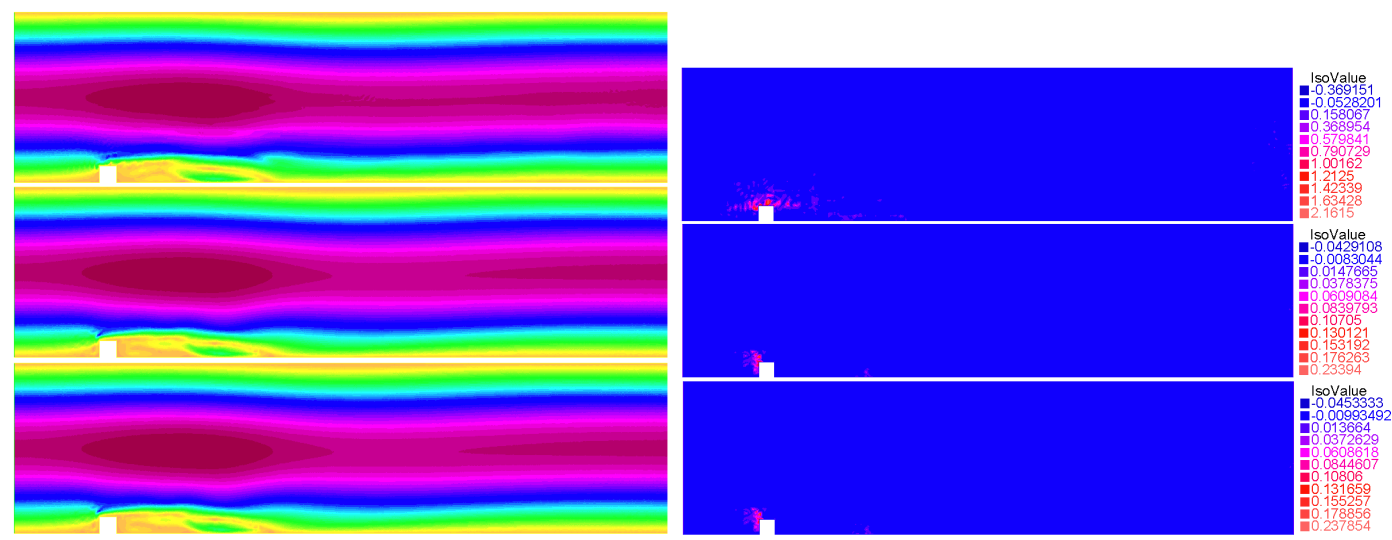

FIG. 5.2. Flow speed and divergence contours at time $t=40$ for Non-Stabilized (top), Standard Stabilized (middle) and BDF2-mgd (down) with $\gamma=1, \beta=0$.

$\beta=0$. Drag $c_{d}(t)$ and lift $c_{l}(t)$ coefficients are calculated; maximum values are presented in Table 5.4. The pressure difference between the front and back of the cylinder $(\Delta p(t)=$ $p(0.15,0.2, t)-p(0.25,0.2, t))$ and both the $L^{2}\left(0, T ; L^{2}(\Omega)\right)$ and $L^{\infty}\left(0, T ; L^{2}(\Omega)\right)$ norms of the velocity divergence are also tabulated in Table 5.4. Furthermore, Figure 5.3 shows velocity speed and vectors for BDF2-mgd at times $t=4,6,7,8$, which are consistent with that in 2, 5, 13, 21.

In Table 5.4, we see that grad-div stabilization effectively reduces the divergence error, as expected. This results in improved accuracy of Standard Stabilized and BDF2-mgd over the Non-Stabilized solution. In particular, both stabilized algorithms produce accurate lift coefficients and smaller divergence errors. 


\begin{tabular}{cccccc}
\hline Method & $c_{d}^{\max }$ & $c_{l}^{\max }$ & $\Delta p_{h}^{N}$ & $\left|\left\|\nabla \cdot u_{h} \mid\right\|_{2,0}\right.$ & $\left\|\nabla \cdot u_{h}^{N}\right\|$ \\
\hline Non-Stabilized & 2.950 & $\mathbf{0 . 4 4 1}$ & -0.1084 & $\mathbf{1 . 9 6 7}$ & $\mathbf{0 . 1 8 6}$ \\
Standard Stabilized & 2.950 & 0.477 & -0.1115 & 0.859 & 0.072 \\
BDF2-mgd & 2.950 & 0.475 & -0.1115 & 0.906 & 0.074 \\
\hline
\end{tabular}

Maximum lift, drag coefficients, pressure drop, and divergence quantities for flow past a cylinder.
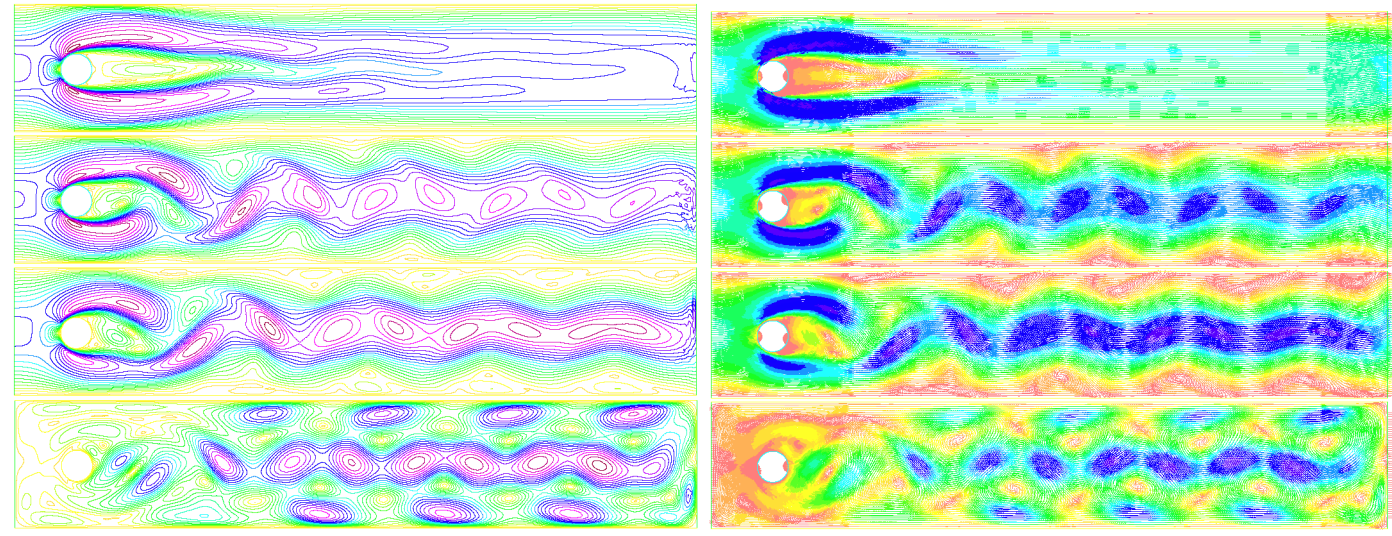

FIG. 5.3. Flow speed and vectors for flow past a cylinder at times $t=4,6,7$, and 8.

6. Conclusion. We developed a BDF2 time-discrete, modular grad-div stabilization algorithm (BDF2-mgd) for the time dependent Navier-Stokes equations. Compared with methods implementing standard grad-div stabilization, our algorithm produces consistent numerical approximations while avoiding solver breakdown for large grad-div parameters. We prove that this algorithm is unconditionally, nonlinearly, energy stable and second-order accurate in time. Numerical tests illustrate the theoretical results and computational efficiency.

To impose discrete versions of $-\beta \nabla \nabla \cdot u_{t}-\gamma \nabla \nabla \cdot u$, modular grad-div requires a solve of the form $\left(\frac{1}{\Delta t} I+\left(\frac{\beta}{\delta t}+\gamma\right) G\right) u=R H S$, where $G$ is the symmetric positive semi-definite graddiv matrix. For constant $\Delta t$, efficiency increases can exploit the fact that the matrix is fixed. For variable timestep and $\beta=0$, the matrix is a variable shift of $G$ and efficient algorithms exist exploiting this structure. Important next steps include investigating, analytically, the $\nu$ dependence of $\nu^{-1} \inf _{q_{h} \in Q_{h}}\left|\left\|p-p_{h}\right\|\right|_{2,0}^{2}$ in Theorems 4.4 and 4.5 extending these results to alternative numerical methods, and including sparse, effective variants of grad-div stabilization.

\section{REFERENCES}

[1] R.A. Adams, Sobolev spaces, Academic press, New York, 1995.

[2] A. L. Bowers, S. Le Borne, and L. G. Rebholz, Error analysis and iterative solvers for Navier-Stokes projection methods with standard and sparse grad-div stabilization, Comput. Methods Appl. Mech. Engrg., 275 (2014), pp. 1-19.

[3] V. DeCaria, W. Layton, and M. McLaughlin, A conservative, second order, unconditionally stable artificial compression method, Comput. Methods Appl. Mech. Engrg., 325 (2017), pp. 733-747.

[4] V. DeCaria, W. J. Layton, A. Pakzad, Y. Rong, N. Sahin, and H. Zhao, On the determination of the grad-div criterion, Apr. 2017, https://arxiv.org/abs/1704.04171.

[5] J. A. Fiordilino, W. J. Layton, and Y. Rong, Robust and Efficient Modular Grad-Div Stabilization, Comput. Methods Appl. Mech. Engrg., 335 (2018), pp. 327-346.

[6] V. P. Fragos, S. P. Psychoudaki, and N. A. Malamataris, Computer-aided analysis of flow past a surfacemounted obstacle, Int. J. Numer. Meth. Fluids, 25 (1997), pp. 495-512.

[7] V. Girault and P. A. Raviart, Finite Element Approximation of the Navier-Stokes Equations, Springer, 
Berlin, 1979.

[8] R. Glowinski and P. Le Tallec, Augmented Lagrangian and operator-splitting methods in nonlinear mechanics, SIAM, Philadelphia, 1989.

[9] J.-L. Guermond and P. D. Minev, High-order time stepping for the Navier-Stokes equations with minimal computational complexity, Journal of Computational and Applied Mathematics, 310 (2017), pp. 92103.

[10] T. Heister and G. Rapin, Efficient augmented Lagrangian-type preconditioner for the Oseen problem using grad-div stabilization, Int. J. Numer. Meth. Fluids, 71 (2013), pp. 118-134.

[11] J. G. Heywood and R. Rannacher, Finite-Element Approximation of the Nonstationary Navier-Stokes Problem Part IV: Error Analysis for Second-Order Time Discretization, SIAM J. Numer. Anal., 27 (1990), pp. 353-384.

[12] E. W. Jenkins, V. John, A. Linke, and L. G. Rebholz, On the parameter choice in grad-div stabilization for the Stokes equations, Adv. Comput. Math., 40 (2014), pp. 491-516.

[13] V. John, Reference values for drag and lift of a two-dimensional time-dependent flow around a cylinder, Int. J. Numer. Meth. Fluids, 44 (2004), pp. 777-788.

[14] V. John and A. Liakos, Time-dependent flow across a step: the slip with friction boundary condition, Int. J. Numer. Meth. Fluids, 50 (2006), pp. 713-731.

[15] V. John, A. Linke, C. Merdon, M. Neilan, and L. G. Rebholz, On the Divergence Constraint in Mixed Finite Element Methods for Incompressible Flows, SIAM Review, 59 (2017), pp. 492-544.

[16] L. P. Franca and T. J. Hughes, Two classes of mixed finite element methods, Computer Methods in Applied Mechanics and Engineering, 69.1 (1988), pp. 89-129.

[17] W. Layton, Introduction to the Numerical Analysis of Incompressible, Viscous Flows, SIAM, Philadelphia, 2008.

[18] W. J. Layton and L. G. Rebholz, On relaxation times in the Navier-Stokes-Voigt model, International Journal of Computational Fluid Dynamics, 27 (2013), pp. 184-187.

[19] W. Layton and L. Tobiska, A Two-Level Method with Backtracking for the Navier-Stokes Equations, SIAM J. Numer. Anal., 35 (1998), pp. 2035-2054.

[20] W. Layton, C. C. Manica, M. Neda, M. Olshanskii, and L. G. Rebholz, On the accuracy of the rotation form in simulations of the Navier-Stokes equations, Journal of Computational Physics, 228 (2009), pp. 3433-3447.

[21] A. Linke and L. G. Rebholz, On a reduced sparsity stabilization of grad-div type for incompressible flow problems, Comput. Methods Appl. Mech. Engrg., 261-262 (2013), pp. 142-153.

[22] A. Linke, L. G. Rebholz, and N. E. Wilson, On the convergence rate of grad-div stabilized Taylor-Hood to Scott-Vogelius solutions for incompressible flow problems, Journal of Mathematical Analysis and Applications., 381 (2011), pp. 612-626.

[23] G. Lube and M. A. Olshanskii, Stable finite-element calculation of incompressible flows using the rotation form of convection, IMA Journal of Numerical Analysis, 22 (2002), pp. 437-461.

[24] Y. Rong and Y. Hou, A partitioned second-order method for magnetohydrodynamic flows at small magnetic reynolds numbers, Numerical Methods for Partial Differential Equations, 33 (2017), pp. 1966-1986.

[25] S. Le Borne and L. Rebholz, Preconditioning sparse grad-div/augmented Lagrangian stabilized saddle point systems, Computing and Visualization in Science, 16 (2015), pp. 259-269.

[26] M. A. Olshanskii, A low order Galerkin finite element method for the Navier-Stokes equations of steady incompressible flow: a stabilization issue and iterative methods, Comput. Methods Appl. Mech. Engrg., 191 (2002), pp. 5515-5536.

[27] M. A. Olshanskii and A. Reusken, Grad-div stabilization for Stokes equations, Mathematics of Computation, 73 (2004), pp. 1699-1718.

[28] M. A. Olshanskii, G. Lube, T. Heister, and J. Löwe, Grad-div stabilization and subgrid pressure models for the incompressible Navier-Stokes equations, Comput. Methods Appl. Mech. Engrg., 198 (2009), pp. 3975-3988.

[29] A. Prohl, On Pressure Approximation via Projection Methods for Nonstationary Incompressible NavierStokes Equations, SIAM J. Numer. Anal., 47 (2008), pp. 158-180.

[30] H. G. Roos, M. Stynes and L. Tobiska, Robust Numerical Methods for Singularly Perturbed Differential Equations: Convection-Diffusion-Reaction and Flow Problems, Springer, Berlin, 2008.

[31] M. Schäfer and S. Turek, Benchmark Computations of Laminar Flow Around a Cylinder, Flow Simulation with High-Performance Computers II, 48 (1996), pp. 547-566.

[32] F. Hecht, New development in FreeFem++, J. Numer. Math., 20 (2012), pp. 251-265. 